\title{
DIFFERENTIATION AND THE BALIAN-LOW THEOREM
}

\author{
John J. Benedetto, Christopher Heil, and David F. Walnut \\ "If it is true, it can be proved." - Enrico Fermi
}

ABSTRACT. The Balian-Low theorem (BLT) is a key result in time-frequency analysis, originally stated by Balian and, independently, by Low, as: If a Gabor system $\left\{e^{2 \pi i m b t} g(t-n a)\right\}_{m, n \in \mathbf{Z}}$ with $a b=1$ forms an orthonormal basis for $L^{2}(\mathbf{R})$, then

$$
\left(\int_{-\infty}^{\infty}|\operatorname{tg}(t)|^{2} d t\right)\left(\int_{-\infty}^{\infty}|\gamma \hat{g}(\gamma)|^{2} d \gamma\right)=+\infty
$$

The BLT was later extended from orthonormal bases to exact frames.

This paper presents a tutorial on Gabor systems, the BLT, and related topics, such as the Zak transform and Wilson bases. Because of the fact that $\left(g^{\prime}\right)^{\wedge}(\gamma)=2 \pi i \gamma \hat{g}(\gamma)$, the role of differentiation in the proof of the BLT is examined carefully. The major new contributions of this paper are the construction of a complete Gabor system of the form $\left\{e^{2 \pi i b_{m} t} g\left(t-a_{n}\right)\right\}$ such that $\left\{\left(a_{n}, b_{m}\right)\right\}$ has density strictly less than 1 , an Amalgam BLT that provides distinct restrictions on Gabor systems $\left\{e^{2 \pi i m b t} g(t-n a)\right\}$ that form exact frames, and a new proof of the BLT for exact frames that does not require differentiation and relies only on classical real variable methods from harmonic analysis.

\section{Introduction}

\subsection{History}

The Balian-Low theorem (BLT) is a key result in time-frequency analysis. It was originally stated by Balian [Bal81] and, independently, by Low [Low85], as: If a Gabor system $\left\{e^{2 \pi i m b t} g(t-\right.$ $n a)\}_{m, n \in \mathbf{Z}}$ with $a b=1$ forms an orthonormal basis for $L^{2}(\mathbf{R})=\left\{f: \int_{-\infty}^{\infty}|f(t)|^{2} d t<\infty\right\}$ then

$$
\left(\int_{-\infty}^{\infty}|\operatorname{tg}(t)|^{2} d t\right)\left(\int_{-\infty}^{\infty}|\gamma \hat{g}(\gamma)|^{2} d \gamma\right)=+\infty
$$

1991 Mathematics Subject Classification. 42-02, 42C15, 42C30.

Key words and phrases. Balian-Low Theorem, Classical Uncertainty Principle Inequality, density of exponentials, frames, Gabor systems, Wilson bases, Zak transform.

Acknowledgements and Notes. The authors were partially supported by the MITRE Sponsored Research Program. The second author was also partially supported by National Science Foundation Grant DMS-9401340.

(C) 1995 CRC Press, Inc.

ISSN 1069-5869 
where $\hat{g}(\gamma)=\int_{-\infty}^{\infty} g(t) e^{-2 \pi i \gamma t} d t$ is the Fourier transform of $g, \mathbf{R}$ is the real line, and $\mathbf{Z}$ is the set of integers. The setting for Balian was Gabor's notion of information area in signal analysis [Gab46], [Dau86], [JHJ88], [Ben94, §7.3], as well as the role of the phase plane in quantum mechanics [vN55]. The background for Low included completeness properties of classical wavepackets in quantum mechanics (for example, [vN55] (including von Neumann's work from the late 1920's).

The proofs given by Balian and Low each contained a gap arising from the fact that squareintegrability of the partial derivatives of a function does not imply continuity of the function itself in dimensions two and higher. This gap was independently addressed in two ways. Battle provided an elegant and entirely new proof based on the operator theory associated with the Classical Uncertainty Principle Inequality [Bat88]. Daubechies, Coifman, and Semmes retained the original approach of Balian and Low, filling the gap directly [Dau90]. In the process, they extended the result from Gabor systems which form orthonormal bases for $L^{2}(\mathbf{R})$ to Gabor systems which form exact frames - a natural generalization of orthonormal bases that we describe below. Although Battle's uncertainty principle approach to the BLT for orthonormal bases was both elegant and simple, and although the step from orthonormal bases to exact frames seems small, it was a nontrivial matter to adapt Battle's proof to the case of exact frames. This was accomplished by Daubechies and Janssen in [DJ93]. Their proof is beautifully recounted in Daubechies' recent monograph [Dau92].

In order to describe frames and exact frames, let us first recall and describe orthonormal bases. We begin with the $L^{2}$-norm of a complex-valued function $f$, given by

$$
\|f\|_{2}=\left(\int|f(t)|^{2} d t\right)^{1 / 2}
$$

Integrals with unspecified limits are to be taken over the entire real line $\mathbf{R}$. The quantity $\|f\|_{2}^{2}$ is often referred to as the energy of $f$. Thus $L^{2}(\mathbf{R})$ is the space of all functions $f$ that have finite energy. We can define an inner product on $L^{2}(\mathbf{R})$ by

$$
\langle f, g\rangle=\int f(t) \overline{g(t)} d t
$$

the bar denoting complex conjugation.

A sequence $\left\{f_{k}\right\}$ of functions in $L^{2}(\mathbf{R})$ is orthonormal if

$$
\left\langle f_{k}, f_{\ell}\right\rangle=\delta_{k, \ell}= \begin{cases}1, & \text { if } k=\ell \\ 0, & \text { if } k \neq \ell\end{cases}
$$

A sequence $\left\{f_{k}\right\}$ is a basis for $L^{2}(\mathbf{R})$ if

$$
\forall f \in L^{2}(\mathbf{R}), \exists \text { unique scalars } c_{k}(f) \text { such that } f=\sum_{k} c_{k}(f) f_{k} .
$$

A sequence that is both orthonormal and a basis is an orthonormal basis. In this case, the scalars $c_{k}(f)$ are given simply by inner products of $f$ with the basis elements: $c_{k}(f)=\left\langle f, f_{k}\right\rangle$. Thus, for an orthonormal basis

$$
\forall f \in L^{2}(\mathbf{R}), \quad f=\sum_{k}\left\langle f, f_{k}\right\rangle f_{k} .
$$


Moreover, the Plancherel formula states that the energy of $f$ is determined by the "energy" of the coefficients $\left\langle f, f_{k}\right\rangle$ :

$$
\forall f \in L^{2}(\mathbf{R}), \quad\|f\|_{2}^{2}=\sum_{k}\left|\left\langle f, f_{k}\right\rangle\right|^{2} .
$$

The Plancherel formula motivates the definition of frames. A sequence $\left\{f_{k}\right\}$ is a frame if there exist constants $A, B>0$, called the frame bounds, so that the following approximate Plancherel formula holds:

$$
\forall f \in L^{2}(\mathbf{R}), \quad A\|f\|_{2}^{2} \leq \sum_{k}\left|\left\langle f, f_{k}\right\rangle\right|^{2} \leq B\|f\|_{2}^{2}
$$

Thus, for a frame, the energy $\|f\|_{2}^{2}$ of $f$ is "equivalent" but not necessarily equal to the coefficient energy $\sum\left|\left\langle f, f_{k}\right\rangle\right|^{2}$. If $A=B$ then the frame is said to be tight.

Unlike orthonormal bases, this approximate Plancherel formula is the only requirement we place on a sequence in order for it to be called a frame. We do not require that a frame form a basis, and in fact there are many useful frames that are not bases. This stems from the surprising fact that every frame provides a series representation of functions $f$ similar to the one given in (1.2) for orthonormal bases. In particular, for any frame $\left\{f_{k}\right\}$ there will exist a dual frame $\left\{\tilde{f}_{k}\right\}$ so that

$$
\forall f \in L^{2}(\mathbf{R}), \quad f=\sum_{k}\left\langle f, \tilde{f}_{k}\right\rangle f_{k}=\sum_{k}\left\langle f, f_{k}\right\rangle \tilde{f}_{k}
$$

If the frame $\left\{f_{k}\right\}$ happens to form a basis, then the only way to write $f=\sum c_{k} f_{k}$ is with $c_{k}=\left\langle f, \tilde{f}_{k}\right\rangle$. If the frame is not a basis then there will exist other choices of $c_{k}$ so that $f=\sum c_{k} f_{k}$. Among all these choices, $c_{k}=\left\langle f, \tilde{f}_{k}\right\rangle$ is still "best" in several ways. For example, it is the choice that minimizes the quantity $\sum\left|c_{k}\right|^{2}$.

It is the concept of exactness that distinguishes between frames that are bases and those that are not. A frame $\left\{f_{k}\right\}$ is exact if it ceases to be a frame when any single element $f_{\ell}$ is deleted, that is, $\left\{f_{k}\right\}_{k \neq \ell}$ is not a frame for any $\ell$. A frame is a basis if and only if it is exact. Therefore, an exact frame satisfies both the approximate Plancherel formula (1.4) and has the property that (1.5) is the unique series representation of functions $f$ in terms of the frame elements $f_{k}$ or the dual frame elements $\tilde{f}_{k}$. All orthonormal bases are exact frames, and all exact frames are frames. However, there exist frames that are not exact, and there exist exact frames that are not orthonormal bases. Moreover, tightness and exactness are distinct concepts: there exist tight frames that are exact and there exist tight frames that are inexact. We discuss further properties of frames in $\S 2.1$.

We can now state the BLT for exact frames. For simplicity of notation, we write $g_{p, q}(t)=$ $e^{2 \pi i p t} g(t-q)$. We call $\left\{g_{m b, n a}\right\}$ a Gabor system, and we assume that the indices $m, n$ range over $\mathbf{Z}$ unless otherwise specified. Then the BLT is:

\subsection{Theorem (BLT).}

Let $g \in L^{2}(\mathbf{R})$, and let $a, b>0$ satisfy $a b=1$. If the Gabor system $\left\{g_{m b, n a}\right\}$ is an exact frame for $L^{2}(\mathbf{R})$, then

$$
\|\operatorname{tg}(t)\|_{2}\|\gamma \hat{g}(\gamma)\|_{2}=+\infty
$$


We shall see in $\S 2.3$ that the hypothesis $a b=1$ in the BLT is redundant, following from the hypothesis that the Gabor system $\left\{g_{m b, n a}\right\}$ forms an exact frame for $L^{2}(\mathbf{R})$ (Theorem 2.3). Moreover, when $a b=1$, a Gabor system is a frame if and only if it is an exact frame (Theorem 3.1d).

It is instructive to compare the BLT to the Classical Uncertainty Principle Inequality. We use the symbol $\hat{\mathbf{R}}$ to denote the real line thought of as the frequency axis, and we let $\mathbf{C}$ denote the set of complex numbers.

\subsection{Theorem (Classical Uncertainty Principle Inequality).}

$$
\begin{aligned}
\text { Let }\left(t_{0}, \gamma_{0}\right) \in \mathbf{R} \times \hat{\mathbf{R}} \text {. Then } & \\
& \forall f \in L^{2}(\mathbf{R}), \quad\|f\|_{2}^{2} \leq 4 \pi\left\|\left(t-t_{0}\right) f(t)\right\|_{2}\left\|\left(\gamma-\gamma_{0}\right) \hat{f}(\gamma)\right\|_{2},
\end{aligned}
$$

and there is equality in (1.7) if and only if $f(t)=C e^{2 \pi i t \gamma_{0}} e^{-s\left(t-t_{0}\right)^{2}}$ for some $C \in \mathbf{C}$ and $s>0$.

Thus, if a Gabor system $\left\{g_{m b, n a}\right\}$ forms an exact frame then the right side of (1.7) is infinite when $f$ is replaced by $g$. In this sense, the BLT maximizes the Classical Uncertainty Principle Inequality. For comparison, note that if $f(t)=(\sin 2 \pi \Omega t) /(\pi t)$, or if $f \in L^{2}(\mathbf{R})$ behaves like $|t|^{\alpha}$ as $t \rightarrow \infty$ with $\alpha \in[-3 / 2,-1 / 2)$, then the right side of (1.7) is infinite.

\subsection{Outline}

Because of the fact that

$$
\left(g^{\prime}\right)^{\wedge}(\gamma)=2 \pi i \gamma \hat{g}(\gamma)
$$

(at least for $g$ satisfying certain conditions, for example, [Ben96, Theorem 1.4.1d,e] or Theorem 5.2), it is natural that differentiation plays a role in any study of the BLT. This differentiation may take several forms, including classical, pointwise almost everywhere, or distributional. It is our goal in this paper to examine this role, to consider the form the differentiation takes, and to determine if differentiation is required in any proof of the BLT. In the process we present a tutorial on the BLT and its current proofs, and explore several related phenomena.

We begin in $\S 2$ by presenting background and context for the BLT. We discuss basic properties of frames in $\S 2.1$ and show in $\S 2.2$ that properties of the Gabor system $\left\{g_{m b, n a}\right\}$ are related to the "density" of the set $\Lambda=\{(n a, m b)\}_{m, n \in \mathbf{Z}}=a \mathbf{Z} \times b \mathbf{Z}$, which is a rectangular lattice in $\mathbf{R} \times \hat{\mathbf{R}}$. Small values of $a, b$ correspond to high density for $\Lambda$, while large values of $a, b$ correspond to low density. We see that the condition $a b=1$, corresponding to $\Lambda$ with density 1 , can be interpreted as a Nyquist phenomenon for Gabor systems: a Gabor system can be a frame only when $a b \leq 1$, can be an exact frame only when $a b=1$, and must be incomplete if $a b>1$. Completeness and related concepts are defined in $\S 2.1$. In $\S 2.3$ we turn from considering such "regular" Gabor systems to "irregular" Gabor systems $\left\{g_{p, q}\right\}_{(q, p) \in \Lambda}$, where $\Lambda$ is now a discrete subset of $\mathbf{R} \times \hat{\mathbf{R}}$, which need not form a lattice. We define the density of such irregular sets, and see that properties of irregular Gabor systems are again tied to the density of $\Lambda$. One of the contributions of this paper is our construction in $\S 2.4$ of an irregular Gabor system that violates the Nyquist phenomenon observed by regular Gabor systems. Specifically, for each integer $K>0$ we show that there exists an irregular Gabor system $\left\{g_{p, q}\right\}_{(q, p) \in \Lambda}$ that is complete in $L^{2}(\mathbf{R})$ even though $\Lambda$ has density $2 / K$. 
In $\S 3$ we develop and apply the Zak transform, a major tool in the analysis of Gabor systems. The basic definitions and properties of the Zak transform are laid out in $\S 3.1$. We use the Zak Transform in $\S 3.2$ to prove a variation on the BLT that we call the Amalgam BLT. This uses the same hypotheses as the BLT and gives a related but distinct conclusion. The Amalgam BLT has the advantage of possessing an elegantly simple proof that applies both to orthonormal bases and to exact frames. We provide examples illustrating the distinction between the BLT and the Amalgam BLT. In $\S 3.3$ we show that these examples provide insight into the class of Gabor systems that forms Bessel sequences, i.e., Gabor systems that satisfy at least the upper frame bound. In $\S 3.4$ we discuss discrete analogues of Gabor systems and the Zak transform and compare BLT phenomena in the continuous and discrete settings.

The BLT places a severe restriction on those functions $g$ such that $\left\{g_{m b, n a}\right\}$ can form an exact frame. Wilson bases were originated as a way to avoid this restriction. They are constructed from linear combinations of Gabor system elements. This seemingly small modification permits Wilson bases to avoid the BLT phenomenon. We devote $\S 4$ to a discussion of Wilson bases. We show how they are related to Gabor systems for which $\Lambda=\{(n a, m b)\}$ has density $1 / 2$ and discuss how they can be analyzed using the Zak transform.

We set the stage for the BLT itself in $\S 5$ by presenting a number of elementary but revealing calculations, as well as placing the BLT in the context of some major ideas from classical harmonic analysis. In the category of calculations there is a comparison of distributional and ordinary differentiation (Theorem 5.1), a quantitative statement about equation (1.8) (Theorem 5.2), and an assortment of facts dealing with the BLT as related to types of bounded variation arising in classical harmonic analysis (Example 5.3). In the category of context, we prove elementary versions of the BLT using Sobolev's Lemma (Remark 5.4) and Wiener amalgam spaces (Theorem 5.5 and Theorem 5.6).

Another contribution of this paper appears in $\S 6$, which is devoted to the Coifman-SemmesDaubechies approach to the BLT. We present a new proof of the key steps using only real variable differentiation methods from harmonic analysis, e.g., [Ste70], combined with the topological arguments from Balian and Low. This represents an attempt to understand the role of differentiation in the BLT in terms of inequalities related to variations of the Zak transform over small cubes.

Finally, in $\S 7$ we consider the BLT from Battle's uncertainty principle point of view. We elaborate on the proof of the BLT for exact frames given by Daubechies-Janssen. This proof is based on the operator theory associated with the Classical Uncertainty Principle Inequality, which is stated in Theorem 1.2 and restated in operator-theoretic terms in Theorem 7.1. After proving two lemmas related to Battle's original idea, we prove a Weak BLT (Theorem 7.4). This result is proved by Hilbert space methods alone, but has the feature of highlighting the role of the position operator $\operatorname{Pf}(t)=t f(t)$ and the dual function $\tilde{g}$ in determining if the Gabor system $\left\{g_{m b, n a}\right\}$ is an exact frame. Battle's BLT for orthonormal bases is a consequence of Theorem 7.4, as is the BLT for several other Gabor systems (Example 7.5). Further, Theorem 7.4 coupled with a differentiation argument gives another proof of the BLT for exact frames (Theorem 7.7). The remainder of $\S 7$ deals with examples closely related to the relation between differentiation and the BLT. We isolate the key step at which Hilbert space methods appear to fail to complete the proof of the BLT for exact frames. We close with an open problem related to this key step, and with the observation that a solution of this problem without the use of differentiation would yield a proof of the BLT in which differentiation plays no role.

We use the usual notation of analysis as found in the books by Hörmander [Hör83], Schwartz [Sch66], and Stein and Weiss [StW71]. For the convenience of the reader we have collected in the Appendix a summary of the various notation used throughout the paper. 


\section{Background}

\subsection{Frames}

Frames can be defined in any Hilbert space, of which $L^{2}(\mathbf{R})$ is a prime example. We let $H$ denote any Hilbert space with inner product $\langle f, g\rangle$ and norm $\|f\|=\langle f, f\rangle^{1 / 2}$. A sequence $\left\{f_{k}\right\}$ in $H$ is a frame for $H$ if there exist constants $A, B>0$ such that the approximate Plancherel formula (1.4) holds with $L^{2}(\mathbf{R})$ replaced by $H . A, B$ are the frame bounds. The frame is tight if $A=B$, and it is exact if it is no longer a frame when any one of its elements is removed. A sequence that satisfies the upper frame bound (and that may or may not satisfy the lower frame bound) is called a Bessel sequence.

The theory of frames is due to Duffin and Schaeffer [DS52], whose context was the theory of nonharmonic Fourier series. Frame analysis has seen a recent resurgence in both time-frequency analysis and wavelet theory, spurred by the papers [DGM86] and [Dau90]. Expository treatments of frames can be found in [HW89], [Dau92], and [BW94]. Gröchenig [Grö91] determined the nontrivial extension of frames to Banach spaces and developed applications to atomic decompositions. Recent developments in pure frame theory can be found in [CH94], [Grö93], [Hol94], [Li94]. There are recent applications of frames in signal processing, for example, [BT93], [HRT94].

A frame may or may not be a basis for $H$. A basis is a sequence $\left\{f_{k}\right\}$ such that (1.1) holds with $L^{2}(\mathbf{R})$ replaced by $H$. Every basis is complete, that is, the finite linear span

$$
\operatorname{span}\left\{f_{k}\right\}=\left\{\sum_{k \in F} c_{k} f_{k}: F \text { is finite, } c_{k} \in \mathbf{C}\right\}
$$

is dense in $H$, meaning that for every $f \in H$ and every $\varepsilon>0$, there is some finite linear combination $h=\sum_{k \in F} c_{k} f_{k}$ so that $\|f-h\|<\varepsilon$. Thus the closure of $\operatorname{span}\left\{f_{k}\right\}$ is all of $H$. It follows from the Hahn-Banach Theorem that $\left\{f_{k}\right\}$ is complete if and only if

$$
\left\langle f, f_{k}\right\rangle=0 \text { for all } k \Longrightarrow f=0 .
$$

Using this formulation, we see immediately from the approximate Plancherel formula (1.4) that all frames are complete.

A basis need not be a frame, and a frame need not be a basis. All exact frames are bases. In fact, an exact frame $\left\{f_{k}\right\}$ is a bounded unconditional basis. Bounded means that $0<\inf \left\|f_{k}\right\| \leq$ $\sup \left\|f_{k}\right\|<\infty$. Unconditional means that the summations in (1.1) converge unconditionally, that is, regardless of the order of summation, so every rearrangement of the series also converges. Conversely, every bounded unconditional basis for $H$ is an exact frame for $H$. Exact frames can be characterized in yet another way: They coincide with the class of Riesz bases for $H$. A sequence $\left\{f_{k}\right\}$ is a Riesz basis if there exists a continuous, invertible, linear mapping $T$ of $H$ onto itself such that $\left\{T f_{k}\right\}$ forms an orthonormal basis for $H$. Thus the notions of exact frame, bounded unconditional basis, and Riesz basis coincide. Proofs of these equivalences can be found in [You80], and are due to Köthe [Köt36] and Duffin and Schaeffer [DS52], with important related contributions by Bari [Bar51].

Whether it forms a basis or not, any frame $\left\{f_{k}\right\}$ allows series representations of elements $f \in H$ : There will exist coefficients $c_{k}(f)$ so that $f=\sum c_{k}(f) f_{k}$. If the frame is not exact then these coefficients need not be unique. However, there is always one computable, canonical choice. This is derived from the frame operator 


$$
S f=\sum_{k}\left\langle f, f_{k}\right\rangle f_{k}
$$

which is a continuous, invertible, linear mapping of $H$ onto itself. In fact, $\left\|I-\frac{2}{A+B} S\right\|<1$, so $S^{-1}$ can be computed via the Neumann series $S^{-1}=\frac{2}{A+B} \sum_{k=0}^{\infty}\left(I-\frac{2}{A+B} S\right)^{k}$. The sequence $\left\{\tilde{f}_{k}\right\}$ with $\tilde{f}_{k}=S^{-1} f_{k}$ forms another frame for $H$, called the dual frame of $\left\{f_{k}\right\}$. The frame bounds for the dual frame are $1 / B, 1 / A$. Using the dual frame we obtain the series representations given in (1.5), which express $f$ in terms of the frame elements $f_{k}$. The summations in (1.5) converge unconditionally. If the frame $\left\{f_{k}\right\}$ is exact, then $\left\{f_{k}\right\}$ and the dual frame $\left\{\tilde{f}_{k}\right\}$ are biorthogonal, that is, $\left\langle f_{k}, \tilde{f}_{\ell}\right\rangle=\delta_{k, \ell}$. Conversely, any frame that is biorthogonal to its dual frame is exact.

The frame operator $S$ is a positive operator, that is, $\langle S f, f\rangle \geq 0$ for every $f$. It therefore possesses a square-root $S^{1 / 2}$, a positive, invertible operator satisfying $S^{1 / 2} S^{1 / 2}=S$. The sequence $\left\{S^{-1 / 2} f_{k}\right\}$ is a tight frame with $A=B=1$. This frame is exact if and only if the original frame $\left\{f_{k}\right\}$ is exact.

\subsection{Gabor systems, Gabor frames, and density}

We define the translation $\tau_{x} f$ and modulation $e_{\gamma} f$ of a function $f$ by

$$
\tau_{x} f(t)=f(t-x) \text { and } \quad e_{\gamma} f(t)=e^{2 \pi i \gamma t} f(t) .
$$

Given $g \in L^{2}(\mathbf{R})$ and fixed $a, b>0$, the Gabor system corresponding to $g, a$, and $b$ is the sequence $\left\{g_{m b, n a}\right\}_{m, n \in \mathbf{Z}}$, where

$$
g_{p, q}(t)=e_{p} \tau_{q} g(t)=e^{2 \pi i p t} g(t-q) .
$$

Gabor systems are sometimes referred to as Weyl-Heisenberg or windowed Fourier systems.

A Gabor system that forms a frame for $L^{2}(\mathbf{R})$ is called a Gabor frame. The dual frame of a Gabor frame has a particularly nice form, which follows from the calculation $S\left(g_{m b, n a}\right)=(S g)_{m b, n a}$, where $S$ is the frame operator

$$
S f=\sum_{m, n}\left\langle f, g_{m b, n a}\right\rangle g_{m b, n a}
$$

Replacing $g$ by $S^{-1} g$ gives $S^{-1}\left(g_{m b, n a}\right)=\left(S^{-1} g\right)_{m b, n a}$, so the dual frame of $\left\{g_{m b, n a}\right\}$ is another Gabor frame $\left\{\tilde{g}_{m b, n a}\right\}$, generated by the dual function $\tilde{g}=S^{-1} g$.

The frame operator $S$ and the dual Gabor frame play a key role in understanding and implementing Gabor analysis. Later in this issue of the Journal of Fourier Analysis and Applications there is a paper of Janssen [Jan95] that explores these topics in detail. Other related papers include [Jan94], [Li94], [LH95], [QCL92], [RoS94], [TO95], [WR90], [ZZ93].

The algebraic structure of the lattice $\Lambda=\{(n a, m b)\}=a \mathbf{Z} \times b \mathbf{Z}$ has been exploited to derive necessary conditions for a Gabor system $\left\{g_{m b, n a}\right\}$ to be complete, a frame, or an exact frame, in terms of the value of the product $a b$. It is the product rather than the individual values of $a$ and $b$ that are important because if $h(t)=r^{1 / 2} g(r t)$ is a dilation of $g$ then $h_{p, q}(t)=r^{1 / 2} g_{p / r, q r}(r t)$. Hence the Gabor system $\left\{h_{m b, n a}\right\}$ is complete, a frame, or an exact frame if and only if the same is true of the Gabor system $\left\{g_{m b / r, n a r}\right\}$. 
As a corollary of other results on $C^{*}$ algebras, Rieffel [Rie81] proved that $\left\{g_{m b, n a}\right\}$ is incomplete for any $g$ if $a b>1$. This can be viewed as a Nyquist phenomenon for Gabor systems. Rieffel's proof was nonconstructive, following from the value of the coupling constant of the von Neumann algebra generated by the operators $\left\{e_{m b} \tau_{n a}\right\}$. Daubechies [Dau90] proved Rieffel's theorem without recourse to operator algebras for the special case that $a b$ is rational and exceeds 1 . Her proof exhibited a function orthogonal to each $g_{m b, n a}$. Landau [Lan93] obtained the weaker result that $\left\{g_{m b, n a}\right\}$ cannot be a frame for $L^{2}(\mathbf{R})$ if $a b>1$ and both $g$ and $\hat{g}$ satisfy certain decay conditions. His proof did not rely on the algebraic structure of $\Lambda=a \mathbf{Z} \times b \mathbf{Z}$ and in fact applies to more general, "irregular" $\Lambda$, a topic we explore further in $\S 2.3$. Janssen [Jan94] proved that $\left\{g_{m b, n a}\right\}$ cannot be a frame for any $g \in L^{2}(\mathbf{R})$ if $a b>1$, without restrictions on $g$ or $a b$, but again relying on the algebraic structure of the lattice $a \mathbf{Z} \times b \mathbf{Z}$. In this issue of the Journal of Fourier Analysis and Applications there is a paper of Daubechies, Landau, and Landau [DLL95] that explores the Rieffel incompleteness result in detail, including the connections to operator algebras and to a remarkable result known as the Wexler-Raz identity, which was first stated in [WR90].

\subsection{Irregular Gabor systems}

Landau's work in [Lan93] extended the analysis of Gabor systems from "regular" systems $\left\{g_{m b, n a}\right\}$ where $\Lambda=\{(n a, m b)\}=a \mathbf{Z} \times b \mathbf{Z}$ is a rectangular lattice to "irregular" systems $\left\{g_{p, q}\right\}_{(q, p) \in \Lambda}$ where $\Lambda$ is a discrete subset of $\mathbf{R} \times \hat{\mathbf{R}}$ but not necessarily a lattice. The crucial quantity is still the density of $\Lambda$. We give below a precise definition of density due to Beurling. Density concepts were used by Paley and Wiener [PW34] and by Levinson [Lev40] to study spectral gaps and the uniqueness of nonharmonic Fourier series. Beurling's definition of density was used in the beautiful characterization of complete sets of exponentials due to Beurling and Malliavin [BM62], [BM67], and by Landau in his studies of completeness properties of exponentials [Lan64], and the sampling and interpolation of entire functions [Lan67].

We shall define the density of a subset $\Lambda$ of $d$-dimensional Euclidean space, which we denote by $\mathbf{R}^{d}$ (usually $d=1$ or 2 in this paper). Assume that $\Lambda \subseteq \mathbf{R}^{d}$ is uniformly discrete, that is, there is a minimum separation between the elements of $\Lambda$. Let $B$ denote the ball of volume 1 in $\mathbf{R}^{d}$ centered at the origin. Given a positive $r>0$ and given $x \in \mathbf{R}^{d}$, let $B(x ; r)=\{x+r t: t \in B\}$, so that $B(x ; r)$ is the ball of volume $r^{d}$ centered at $x$. For each $r$, let $\nu^{+}(r)$ and $\nu^{-}(r)$ denote the largest and smallest number of points of $\Lambda$ that lie in any $B(x ; r)$ :

$$
\nu^{+}(r)=\max _{x \in \mathbf{R}^{d}} \#\{\lambda: \lambda \in \Lambda \cap B(x ; r)\} \quad \text { and } \quad \nu^{-}(r)=\min _{x \in \mathbf{R}^{d}} \#\{\lambda: \lambda \in \Lambda \cap B(x ; r)\} .
$$

These quantities are finite because $\Lambda$ is uniformly discrete. The upper and lower uniform Beurling densities of $\Lambda$ are then

$$
D^{+}(\Lambda)=\limsup _{r \rightarrow \infty} \frac{\nu^{+}(r)}{r^{d}} \text { and } D^{-}(\Lambda)=\liminf _{r \rightarrow \infty} \frac{\nu^{-}(r)}{r^{d}} .
$$

Landau has shown that these quantities are unchanged if the ball $B$ is replaced by any other bounded set with measure 1. If $D^{+}(\Lambda)=D^{-}(\Lambda)$ then the set $\Lambda$ is said to have uniform Beurling density $D(\Lambda)=D^{+}(\Lambda)=D^{-}(\Lambda)$.

2.1. Example. Let $d=1$, and consider the rectangular lattice $\Lambda=a \mathbf{Z}=\{a n\}_{n \in \mathbf{Z}}$. Here $B$ is the interval $(-1 / 2,1 / 2)$ with length 1 , so $B(x ; r)=(x-r / 2, x+r / 2)$ has length $r$. When $r>a$, there 
are at least $[r / a]-1$ and at most $[r / a]$ points of $a \mathbf{Z}$ in $B(x ; r)$, where $[t]$ denotes the largest integer not exceeding $t$. Therefore $a \mathbf{Z}$ has uniform Beurling density $D(a \mathbf{Z})=1 / a$.

Now consider the consequences of slightly perturbing $a \mathbf{Z}$. Specifically, suppose that $\Lambda=\left\{\lambda_{n}\right\}$ is such that $\left|\lambda_{n}-a n\right|<\varepsilon$ for every $n$. Then the density is unchanged: $\Lambda$ has uniform Beurling density $D(\Lambda)=1 / a$.

If $\Lambda_{1}$ is a uniformly discrete subset of $\mathbf{R}^{d_{1}}$ and $\Lambda_{2}$ is a uniformly discrete subset of $\mathbf{R}^{d_{2}}$ then $\Lambda_{1} \times \Lambda_{2}$ is a uniformly discrete subset of $\mathbf{R}^{d_{1}+d_{2}}$, and $D^{+}\left(\Lambda_{1} \times \Lambda_{2}\right)=D^{+}\left(\Lambda_{1}\right) D^{+}\left(\Lambda_{2}\right)$. Similar calculations hold for the lower uniform densities. In particular, the density of the lattice $a \mathbf{Z} \times b \mathbf{Z}$ in $\mathbf{R} \times \hat{\mathbf{R}}$ is $D(a \mathbf{Z} \times b \mathbf{Z})=1 /(a b)$.

Landau proved in [Lan93] that if $g \in L^{2}(\mathbf{R})$ satisfies $|g(t)| \leq C /\left(1+|t|^{\alpha}\right)$ and $|\hat{g}(\gamma)| \leq$ $C /\left(1+|\gamma|^{\alpha}\right)$ for some $\alpha>1$, then $\left\{g_{p, q}\right\}_{(q, p) \in \Lambda}$ cannot be a frame if $D(\Lambda)<1$. Landau's proof relied on a phase-space localization theorem of Daubechies [Dau90]. Ramanathan and Steger [RaS95] recently improved Landau's result to apply to all $g \in L^{2}(\mathbf{R})$. Their proof relied on a simple dimension counting technique. Moreover, if $\Lambda$ actually forms a rectangular lattice, their technique recovers the full Rieffel incompleteness result. Specifically, they proved the following theorem [RaS95].

\subsection{Theorem.}

Let $g \in L^{2}(\mathbf{R})$, and let $\Lambda \subseteq \mathbf{R} \times \hat{\mathbf{R}}$ be a uniformly discrete set.

(a) If $D^{+}(\Lambda)<1$, then $\left\{g_{p, q}\right\}_{(q, p) \in \Lambda}$ is not a frame for $L^{2}(\mathbf{R})$.

(b) If $\Lambda=a \mathbf{Z} \times b \mathbf{Z}$ is a rectangular lattice with uniform Beurling density $D(\Lambda)=1 /(a b)<1$, then $\left\{g_{m b, n a}\right\}$ is incomplete in $L^{2}(\mathbf{R})$.

Ramanathan and Steger conjectured in [RaS95] that Theorem 2.2 can be further improved to say that $\left\{g_{p, q}\right\}_{(q, p) \in \Lambda}$ must be incomplete when $\Lambda$ is a uniformly discrete set with $D^{+}(\Lambda)<1$. We show in $\S 2.4$ that this conjecture is false. Thus Theorem 2.2 is the best possible result.

From Theorem 2.2, we see that a Gabor system can only be a frame when the upper uniform Beurling density of $\Lambda$ is at least 1 . In the case of a rectangular lattice $\Lambda=a \mathbf{Z} \times b \mathbf{Z}$ it is easy to see that if the density $1 /(a b)$ equals 1 , i.e., $a b=1$, then $\left\{g_{m b, n a}\right\}$ will be exact (Theorem $3.1 \mathrm{~d}$ ). Ramanathan and Steger proved that density 1 is also a necessary condition for a Gabor system to be a Riesz basis [RaS95], as follows.

\subsection{Theorem.}

Let $g \in L^{2}(\mathbf{R})$, and let $\Lambda \subseteq \mathbf{R} \times \hat{\mathbf{R}}$ be a uniformly discrete set. If $\Lambda$ has uniform Beurling density $D(\Lambda)$ and if $\left\{g_{p, q}\right\}_{(q, p) \in \Lambda}$ is an exact frame for $L^{2}(\mathbf{R})$, then $D(\Lambda)=1$.

In particular, the hypothesis in the BLT (Theorem 1.1) that $a b=1$ is redundant, being a consequence of the hypothesis that the Gabor system is an exact frame. This fact had not been established prior to [RaS95], even in the case of a rectangular lattice $\Lambda$.

Thus, a Gabor frame with uniform Beurling density exceeding 1 will be inexact. Any inexact frame is overcomplete, that is, some elements of the frame can be deleted without destroying the completeness of the system. Heil, Ramanathan, and Topiwala have investigated the independence of such overcomplete Gabor systems [HRT95]. In particular, they have shown that, for a broad class of $g$, every finite subset of the Gabor system $\left\{g_{p, q}\right\}_{(q, p) \in \Lambda}$ is linearly independent even though the entire Gabor system is not independent. 
Feichtinger and Gröchenig have developed a powerful and general theory that applies not only to Gabor frames but to more general atomic decompositions defined on function spaces [FG89], [Grö91]. For the particular case of Gabor systems it implies that, for a certain class of $g$, the Gabor system $\left\{g_{p, q}\right\}_{(q, p) \in \Lambda}$ will be a frame for $L^{2}(\mathbf{R})$ if the density of $\Lambda$ is high enough.

In the opposite direction, the question of how large $a b$ can be in order for $\left\{g_{m b, n a}\right\}$ to generate a frame has been answered for only a few $g$. In the case of the Gaussian $g(x)=e^{-x^{2}}$, the BLT implies that $g$ cannot generate a frame with $a b=1$. Seip and Wallstén have shown that $g$ does generate a frame for every value of $a b<1$ [Sei92a], [SeW92]. The tractability of the Gaussian case is because the mapping $f \mapsto\left\langle f, g_{p, q}\right\rangle e^{\pi\left(p^{2}+q^{2}\right)}$ is an isometry from $L^{2}(\mathbf{R})$ to a Hilbert space of entire functions on $\mathbf{C}$. This Bargmann transform allows techniques from complex analysis to be brought to bear on the problem. A short survey article, including a discussion of related Bergman transforms for wavelet systems, can be found in [Sei92b].

\subsection{Sparse Gabor systems}

We can summarize the discussion in $\S 2.2$ and $\S 2.3$ as follows. If an irregular Gabor system $\left\{g_{p, q}\right\}_{(q, p) \in \Lambda}$ is a frame for $L^{2}(\mathbf{R})$, then the upper uniform Beurling density $D^{+}(\Lambda)$ satisfies $D^{+}(\Lambda) \geq$ 1. If the frame is exact and $\Lambda$ has uniform Beurling density $D(\Lambda)$, then $D(\Lambda)=1$. If $\Lambda=$ $\{(n a, m b)\}=a \mathbf{Z} \times b \mathbf{Z}$ is a lattice, then the Gabor system is incomplete if $D(\Lambda)<1$. We show in this section that this last statement does not extend to irregular Gabor systems, that is, there exist irregular Gabor systems that are complete in $L^{2}(\mathbf{R})$ yet $\Lambda$ has uniform Beurling density $D(\Lambda)<1$.

Our construction is an easy consequence of Landau's fundamental work in [Lan64] on the completeness of sets of exponentials in $C(S)$ when $S$ is finite union of intervals in $\mathbf{R}$. Here $C(S)$ is the space of all continuous functions $f$ with domain $S$. It is a Banach space when equipped with the $L^{\infty}$ norm $\|f\|_{\infty}=\max _{t \in S}|f(t)|$. Landau proved the following theorem [Lan64].

\subsection{Theorem.}

Fix any $\delta>0$ and let $I_{n}$ denote the interval

$$
I_{n}=\left(n-\left(\frac{1}{2}-\delta\right), n+\left(\frac{1}{2}-\delta\right)\right) .
$$

Let $S$ be any finite union of the intervals $I_{n}$. Then for each $\varepsilon>0$ there exists a symmetric real sequence $\left\{\lambda_{k}\right\}_{k \in \mathbf{Z}}$ with $\left|\lambda_{k}-k\right|<\varepsilon$ such that $\left\{e^{2 \pi i \lambda_{k} t}\right\}_{k \in \mathbf{Z}}$ is complete in $C(S)$.

Now we construct our irregular Gabor system. Fix any $0<\varepsilon<1 / 2$, and also fix any $\delta<1 / 4$. Then the intervals $I_{n}$ defined in (2.5) have length $1-2 \delta>1 / 2$. Choose any integer $K>0$ and define

$$
S=\bigcup_{n=0}^{K-1} I_{n} .
$$

Then by Theorem 2.4, there exists a collection $\Lambda=\left\{\lambda_{k}\right\}$ so that $\left\{e^{2 \pi i \lambda_{k} t}\right\}$ is complete in $C(S)$. Because $C(S)$ is dense in $L^{2}(S)$ and because $\|f\|_{2} \leq|S|^{1 / 2}\|f\|_{\infty}$ for each $f \in C(S)$, the set $\left\{e^{2 \pi i \lambda_{k} t}\right\}$ is complete in $L^{2}(S)$ as well. 
Our Gabor system will be generated by the indicator function of $S$ :

$$
g(x)=\mathbf{1}_{S}(x)= \begin{cases}1, & x \in S \\ 0, & x \notin S\end{cases}
$$

We use the following irregular set of time-frequency shifts:

$$
\Gamma=\left\{\left(K n, \lambda_{m}\right)\right\}_{m, n \in \mathbf{Z}} \cup\left\{\left(K n+\frac{1}{2}, \lambda_{m}\right)\right\}_{m, n \in \mathbf{Z}}=(K \mathbf{Z} \times \Lambda) \cup\left(\left(K \mathbf{Z}+\frac{1}{2}\right) \times \Lambda\right) .
$$

Because each element $\lambda_{k} \in \Lambda$ is within $\varepsilon$ of $k$, the densities of $\Lambda$ and $\Gamma$ are easy to compute.

\subsection{Lemma.}

(a) $\Lambda \subseteq \mathbf{R}$ has uniform Beurling density $D(\Lambda)=1$.

(b) $\Gamma \subseteq \mathbf{R} \times \hat{\mathbf{R}}$ has uniform Beurling density $D(\Gamma)=2 / K$.

Proof. The fact that $\Lambda$ has uniform Beurling density 1 in $\mathbf{R}$ follows from Example 2.1. Therefore $K \mathbf{Z} \times \Lambda$ and $\left(K \mathbf{Z}+\frac{1}{2}\right) \times \Lambda$ each have uniform Beurling density $1 / K$ in $\mathbf{R} \times \hat{\mathbf{R}}$. Since these two sets are simply shifts of each other, their union has uniform Beurling density $2 / K$. $L^{2}(\mathbf{R})$.

Finally, we show that the irregular Gabor system generated by $g=\mathbf{1}_{S}$ and $\Gamma$ is complete in

\subsection{Theorem.}

The irregular Gabor system

$$
\left\{g_{p, q}\right\}_{(q, p) \in \Gamma}=\left\{g_{\lambda_{m}, K n}\right\}_{m, n \in \mathbf{Z}} \cup\left\{g_{\lambda_{m}, K n+\frac{1}{2}}\right\}_{m, n \in \mathbf{Z}}
$$

is complete in $L^{2}(\mathbf{R})$.

Proof. Suppose that $f \in L^{2}(\mathbf{R})$ satisfies $\left\langle f, g_{\lambda_{m}, K n}\right\rangle=0$ and $\left\langle f, g_{\lambda_{m}, K n+\frac{1}{2}}\right\rangle=0$ for every $m, n \in \mathbf{Z}$. Fix any $n$. Then for each $m$, we have

$$
\begin{aligned}
0=\left\langle f, g_{\lambda_{m}, K n}\right\rangle & =\int f(t) e^{-2 \pi i \lambda_{m} t} \mathbf{1}_{S}(t-K n) d t \\
& =e^{-2 \pi i \lambda_{m} K n} \int_{S} f(t+K n) e^{-2 \pi i \lambda_{m} t} d t
\end{aligned}
$$

However, $\left\{e^{2 \pi i \lambda_{k} t}\right\}$ is complete in $L^{2}(S)$, so, by (2.1), this implies that $f(t+K n)=0$ for a.e. $t \in S$. Similarly, $f\left(t+K n+\frac{1}{2}\right)=0$ for a.e. $t \in S$. Since the shifts of $S$ by $K n$ and $K n+\frac{1}{2}$ cover the entire line, we conclude that $f=0$ a.e. on $\mathbf{R}$. Therefore, the Gabor system in (2.6) is complete in $L^{2}(\mathbf{R})$. 

small.

By taking $K$ as large as we like, we can make the density of the Gabor system in (2.6) arbitrarily

\section{The Zak Transform and the Amalgam BLT}

\subsection{The Zak Transform}

The Zak transform of a function $f \in L^{2}(\mathbf{R})$ is formally defined by

$$
Z f(t, \omega)=F(t, \omega)=\sum_{k \in \mathbf{Z}} f(t+k) e^{2 \pi i k \omega}, \quad(t, \omega) \in \mathbf{R} \times \hat{\mathbf{R}} .
$$

$Z f$ satisfies the quasiperiodicity relations

$$
Z f(t+1, \omega)=e^{-2 \pi i \omega} Z f(t, \omega) \text { and } Z f(t, \omega+1)=Z f(t, \omega),
$$

and therefore $Z f$ is determined by its values on the cube $Q=[0,1) \times[0,1)$. As with many useful concepts, the Zak transform has been rediscovered many times, and also goes by the name WeilBrezin transform and $k-q$ transform. For properties and history of the Zak transform we refer to [AT75], [DGM86], [HW89], [Jan82], [Jan88], [Zak75].

The Zak transform is a unitary mapping of $L^{2}(\mathbf{R})$ onto $L^{2}(Q)$, where

$$
L^{p}(Q)=\left\{F:\|F\|_{p}=\left(\iint_{Q}|F(t, \omega)|^{p} d t d \omega\right)^{1 / p}<\infty\right\} .
$$

The utility of the Zak transform arises from its action on a Gabor system $\left\{g_{m, n}\right\}$ constructed with $a=b=1$ :

$$
Z\left(g_{m, n}\right)(t, \omega)=e^{2 \pi i m t} e^{2 \pi i n \omega} Z g(t, \omega)=e_{m}(t) e_{n}(\omega) Z g(t, \omega),
$$

where $e_{\gamma}(t)=e^{2 \pi i \gamma t}$. The Zak transform is particularly useful in proving the BLT. Although the BLT is stated for Gabor systems satisfying $a b=1$, the discussion in $\S 2.2$ shows that only the value of the product $a b$ is important; therefore, we may always reduce to the case $a=b=1$.

Consider the Gabor system $\left\{g_{m, n}\right\}$ generated by $g=\mathbf{1}_{[0,1)}$, the indicator function of the interval $[0,1)$. This Gabor system forms an orthonormal basis for $L^{2}(\mathbf{R})$. Note that $g$ satisfies (1.6) since $\|\gamma \hat{g}(\gamma)\|_{2}=\infty$, although $\|t g(t)\|_{2}<\infty$. The Zak transform of $g$ is $Z g(t, \omega)=1$ for $(t, \omega) \in Q$. Therefore $Z g_{m, n}(t, \omega)=e_{m}(t) e_{n}(\omega)$. Thus the Zak transform maps the orthonormal basis $\left\{g_{m, n}\right\}$ for $L^{2}(\mathbf{R})$ onto the orthonormal basis $\left\{e_{m}(t) e_{n}(\omega)\right\}$ for $L^{2}(Q)$. This is one method of verifying that $Z$ is a unitary mapping of $L^{2}(\mathbf{R})$ onto $L^{2}(Q)$.

For general $g \in L^{2}(\mathbf{R})$, the determination of properties of $\left\{g_{m, n}\right\}$ reduces by the Zak transform to the determination of properties of $\left\{e_{m}(t) e_{n}(\omega) Z g\right\}$, which is formed by multiplying each element of the orthonormal basis $\left\{e_{m}(t) e_{n}(\omega)\right\}$ by the single function $Z g$. We expect then that the behavior of $Z g$ will be crucial. Parts a, c, d, and e of the following result are well-known, and a proof is given in [HW89]. A proof of Theorem 3.1b can be found in [Hei90]. 


\subsection{Theorem.}

Let $g \in L^{2}(\mathbf{R})$, and set $a=b=1$. Then

(a) $\left\{g_{m, n}\right\}$ is complete in $L^{2}(\mathbf{R})$ if and only if $Z g \neq 0$ a.e.

(b) $\left\{g_{m, n}\right\}$ is minimal and complete in $L^{2}(\mathbf{R})$ if and only if $1 /(Z g) \in L^{2}(Q)$.

(c) $\left\{g_{m, n}\right\}$ is a Bessel sequence for $L^{2}(\mathbf{R})$ if and only if $Z g \in L^{\infty}(Q)$.

(d) $\left\{g_{m, n}\right\}$ is a frame for $L^{2}(\mathbf{R})$ with frame bounds $A, B$ if and only if

$$
0<A \leq|Z g(t, \omega)|^{2} \leq B<\infty \text { a.e. }
$$

In this case, $\left\{g_{m, n}\right\}$ is an exact frame for $L^{2}(\mathbf{R})$.

(e) $\left\{g_{m, n}\right\}$ is an orthonormal basis for $L^{2}(\mathbf{R})$ if and only if $|Z g(t, \omega)|^{2}=1$ a.e.

The equivalences in Theorem 3.1 fail for Gabor systems with $a b \neq 1$.

The Zak transform has the remarkable property that if $Z g$ is continuous on all of $\mathbf{R} \times \hat{\mathbf{R}}$ (not just on the subset $Q$ ) then $Z g$ has a zero in $Q$. A proof can be found in [HW89, Theorem 4.3.4]. For original proofs and related results, see [AT75, p. 18], [BGZ75], [BZ81], [Jan82], [Zak75]. In light of Theorem 3.1, the continuity - or lack thereof - of $Z g$ is a major influence on any approach to proving the BLT. Note for example that if $g=\mathbf{1}_{[0,1)}$ then $Z g=1$ on $Q$. However, by quasiperiodicity, $Z g$ is not continuous on $\mathbf{R} \times \hat{\mathbf{R}}$.

The Zak transforms of $g$ and $\hat{g}$ are related by the following formulas (Lemma 6.2):

$$
Z \hat{g}(t, \omega)=e^{-2 \pi i t \omega} Z g(\omega,-t) \quad \text { and } \quad Z g(t, \omega)=e^{-2 \pi i t \omega} Z \hat{g}(-\omega, t) .
$$

\subsection{The Amalgam BLT}

In this section we use the Zak transform to prove a variation of the BLT. This Amalgam BLT is stated in terms of a Wiener amalgam space $W(L, G)$. Amalgam spaces allow us to describe a function in terms of both its local (L) and global $(\mathrm{G})$ behavior. For example,

$$
W\left(L^{p}, \ell^{q}\right)=\left\{f:\|f\|_{W\left(L^{p}, \ell^{q}\right)}=\left(\sum_{k}\left\|f \cdot \mathbf{1}_{[k, k+1]}\right\|_{p}^{q}\right)^{1 / q}<\infty\right\} .
$$

Wiener's original amalgam space is the Segal algebra

$$
W\left(C_{0}, \ell^{1}\right)=\left\{f \in W\left(L^{\infty}, \ell^{1}\right): f \text { is continuous }\right\} .
$$

Clearly $W\left(C_{0}, \ell^{1}\right) \subseteq L^{1}(\mathbf{R}) \cap L^{2}(\mathbf{R}) \cap C_{0}(\mathbf{R})$. We also define

$$
W\left(C_{0}, \ell^{2}\right)=\left\{f \in W\left(L^{\infty}, \ell^{2}\right): f \text { is continuous }\right\} .
$$

General Wiener amalgam spaces $W(L, G)$ are defined in [Fei90], [FS85]. There have been recent applications of Wiener amalgam spaces to Wiener-Plancherel formulas and Gabor theory [BBE89], [Hei90], [Wal89a]. 
If $g \in W\left(L^{p}, \ell^{1}\right)$ then the series defining $Z g$ converges absolutely in $L^{p}(Q)$ :

$$
\begin{aligned}
\|Z g\|_{L^{p}(Q)} & \leq \sum_{k}\left\|g(t+k) e^{2 \pi i k \omega}\right\|_{L^{p}(Q)} \\
& =\sum_{k}\left\|g \cdot \mathbf{1}_{[k, k+1]}\right\|_{L^{p}(\mathbf{R})}=\|g\|_{W\left(L^{p}, \ell^{1}\right)}<\infty .
\end{aligned}
$$

Therefore the Zak transform is a continuous mapping of $W\left(L^{p}, \ell^{1}\right)$ into $L^{p}(Q)$. The case $p=\infty$ leads immediately to the following result due to Heil [Hei90].

\subsection{Theorem (Amalgam BLT).}

Let $g \in L^{2}(\mathbf{R})$, and let $a, b>0$ satisfy $a b=1$. If the Gabor system $\left\{g_{m b, n a}\right\}$ is an exact frame for $L^{2}(\mathbf{R})$, then

$$
g \notin W\left(C_{0}, \ell^{1}\right) \quad \text { and } \quad \hat{g} \notin W\left(C_{0}, \ell^{1}\right) .
$$

Proof. It suffices to consider $a=b=1$. If $g \in W\left(C_{0}, \ell^{1}\right)$ then $g(t+k) e^{2 \pi i k \omega}$ is continuous for every $k$. However, by (3.3), $Z g(t, \omega)=\sum g(t+k) e^{2 \pi i k \omega}$ converges in $L^{\infty}$ norm, that is, uniformly on $Q$. The square $Q=[0,1) \times[0,1)$ can be replaced in this argument by any translate of $Q$, so $Z g$ must in fact be continuous on all of $\mathbf{R} \times \hat{\mathbf{R}}$. Therefore $Z g$ must have a zero, so the Gabor system $\left\{g_{m, n}\right\}$ cannot be a frame. Because $\left(g_{m, n}\right)^{\wedge}=e^{2 \pi i m n} \hat{g}_{-n, m}$, the Gabor system generated by $g$ is a frame if and only if the Gabor system generated by $\hat{g}$ is a frame. Therefore $\hat{g} \notin W\left(C_{0}, \ell^{1}\right)$ as well.

The BLT and the Amalgam BLT may both be qualitatively stated as "if $\left\{g_{m, n}\right\}$ is an exact frame then either $g$ is not smooth or it has poor decay at infinity." However, the two results are distinct. We show in Example 3.3 that there exists a function $g \in L^{2}(\mathbf{R})$ such that $\|t g(t)\|_{2}\|\gamma \hat{g}(\gamma)\|_{2}=\infty$ and $g, \hat{g} \in W\left(C_{0}, \ell^{1}\right)$. This means that the BLT does not imply the Amalgam BLT. We show in Example 3.4 that there exists a function $g \in L^{2}(\mathbf{R})$ such that $g, \hat{g} \notin W\left(C_{0}, \ell^{1}\right)$ and $\|t g(t)\|_{2}\|\gamma \hat{g}(\gamma)\|_{2}<\infty$. This means that the Amalgam BLT does not imply the BLT. Thus Theorem 3.2 is neither a restatement of nor a weak form of the BLT.

3.3. Example. We shall construct a function $g$ such that both $g, \hat{g} \in W\left(C_{0}, \ell^{1}\right)$ while $\|\operatorname{tg}(t)\|_{2}\|\gamma \hat{g}(\gamma)\|_{2}=\infty$. Let $f$ be the "tent function" on $[0,1]$, that is,

$$
f(t)=\max \{1-|2 t-1|, 0\} .
$$

Then $f$ is a continuous, piecewise linear function supported on $[0,1]$. Define

$$
g(t)=\sum_{n=1}^{\infty} n^{-3 / 2} f(t-n)
$$

Then $g \in W\left(C_{0}, \ell^{1}\right)$. Further,

$$
\hat{g}(\gamma)=\sum_{n=1}^{\infty} n^{-3 / 2} e^{-2 \pi i n \gamma} \hat{f}(\gamma)=a(\gamma) \hat{f}(\gamma)=a(\gamma) 2 e^{-\pi i \gamma}\left(\frac{\sin (\pi \gamma / 2)}{\pi \gamma}\right)^{2}
$$


where $a(\gamma)=\sum_{n=1}^{\infty} n^{-3 / 2} e^{-2 \pi i n t}$ is a continuous, periodic function. Therefore $\hat{g} \in W\left(C_{0}, \ell^{1}\right)$ as well. Finally,

$$
\begin{aligned}
\int_{n}^{n+1}|t g(t)|^{2} d t & =n^{-3} \int_{n}^{n+1}|t f(t-n)|^{2} d t \\
& \geq n^{-3} \int_{n}^{n+1}|n f(t-n)|^{2} d t=n^{-1}\|f\|_{2}^{2},
\end{aligned}
$$

so $\|t g(t)\|_{2}^{2}=\sum_{n=1}^{\infty} \int_{n}^{n+1}|t g(t)|^{2} d t \geq \sum_{n=1}^{\infty} n^{-1}\|f\|_{2}^{2}=\infty$.

3.4. Example. We shall construct a function $g(t)$ such that $g \in W\left(C_{0}, \ell^{2}\right) \backslash W\left(C_{0}, \ell^{1}\right), \hat{g} \notin$ $W\left(C_{0}, \ell^{1}\right)$, and $\|t g(t)\|_{2}\|\gamma \hat{g}(\gamma)\|_{2}<\infty$.

For $k$ large, say $k \geq N$, let $b_{k}=k-1 / 2$, and let $a_{k}<b_{k}$ have the properties $b_{k}{ }^{3}-a_{k}{ }^{3} \leq k$ and $b_{k}+\frac{1}{k} \leq a_{k+1}-\frac{1}{k+1}$. Define the continuous functions $g_{k}$ by

$$
g_{k}(t)= \begin{cases}\frac{1}{k \log k}, & t \in\left[a_{k}, b_{k}\right], \\ 0, & t \notin\left[a_{k}-\frac{1}{k}, b_{k}+\frac{1}{k}\right] \\ \text { linear, } & t \in\left[a_{k}-\frac{1}{k}, a_{k}\right] \cup\left[b_{k}, b_{k}+\frac{1}{k}\right]\end{cases}
$$

Define $g=\sum_{k=N}^{\infty} g_{k}$. Direct computations yield

$$
\|t g(t)\|_{2}^{2} \leq \frac{7}{3} \sum_{k=N}^{\infty} \frac{1}{k \log ^{2} k}<\infty \quad \text { and } \quad\left\|g^{\prime}\right\|_{2}^{2}=2 \sum_{k=N}^{\infty} \frac{1}{k \log ^{2} k}<\infty
$$

where $g^{\prime}$ is the classical derivative of $g$, defined except at countably many points. Therefore $\|\operatorname{tg}(t)\|_{2}\|\gamma \hat{g}(\gamma)\|_{2}<\infty$. Also,

$$
\sum_{k=N}^{\infty}\left\|g \cdot \mathbf{1}_{[k, k+1]}\right\|_{\infty}=\sum_{k=N}^{\infty} \frac{1}{k \log k}=\infty
$$

and

$$
\sum_{k=N}^{\infty}\left\|g \cdot \mathbf{1}_{[k, k+1]}\right\|_{\infty}^{2}=\sum_{k=N}^{\infty} \frac{1}{k^{2} \log ^{2} k}<\infty .
$$

Therefore, $g \in W\left(C_{0}, \ell^{2}\right) \backslash W\left(C_{0}, \ell^{1}\right)$.

It remains to show that $\hat{g} \notin W\left(C_{0}, \ell^{1}\right)$. If we did have $\hat{g} \in W\left(C_{0}, \ell^{1}\right)$ then $Z \hat{g}$ would be bounded by (3.3). By (3.2), it would then follow that $Z g$ is bounded. Therefore, it suffices to show that $Z g$ is unbounded.

In fact, we can show that for each $M>0$ there is a set $S=\left[-\frac{1}{2}-2 \varepsilon,-\frac{1}{2}-\varepsilon\right] \times[0, \delta]$ such that $|Z g(t, \omega)| \geq M$ for $(t, \omega) \in S$. Specifically, let $K$ be so large that $\sum_{k=N}^{K}(k \log k)^{-1} \geq 2 M$. 
Now choose $\varepsilon>0$ so small that $2 \varepsilon \leq\left|b_{k}-a_{k}\right|$ for $N \leq k \leq K$, and choose $K^{\prime} \geq K$ so large that $\left|b_{k}-a_{k}+\frac{1}{k}\right|<\varepsilon$ for $k \geq K^{\prime}$. Finally, choose $\delta>0$ so small that $\left|1-e^{2 \pi i k \omega}\right| \leq 1 / 2$ if $\omega \in[0, \delta]$ and $N \leq k \leq K^{\prime}$. It can then be shown that for $(t, \omega) \in S$ we have $g(t+k)=0$ if $k<N$ or $k \geq K^{\prime}$, and $g(t+k)=(k \log k)^{-1}$ if $N \leq k \leq K$. Hence, for $(t, \omega) \in S$,

$$
|Z g(t, \omega)|=\left|\sum_{k=N}^{K^{\prime}} g(t+k) e^{2 \pi i k \omega}\right| \geq \frac{1}{2} \sum_{k=N}^{K^{\prime}}|g(t+k)| \geq \frac{1}{2} \sum_{k=N}^{K} \frac{1}{k \log k} \geq M .
$$

\subsection{Bessel Sequences}

In Example 3.4 we constructed a function $g \in W\left(C_{0}, \ell^{2}\right)$ whose Zak transform $Z g$ was unbounded. Since $g \in W\left(C_{0}, \ell^{2}\right)$, the periodic function $\lambda(t)=\sum|g(t-n)|^{2}$ is bounded above:

$$
\lambda(t)=\sum_{n}|g(t-n)|^{2} \leq \sum_{n}\left\|g \cdot \mathbf{1}_{[n, n+1]}\right\|_{\infty}^{2}=\|g\|_{W\left(C_{0}, \ell^{2}\right)}^{2} \text { a.e. }
$$

In order for the Gabor sequence $\left\{g_{m, n}\right\}$ to form a Bessel sequence for $L^{2}(\mathbf{R})$, it is necessary that $\lambda$ be bounded above (see Theorem 3.5b below). However, by Theorem 3.1c, it is also necessary that $Z g$ be bounded. Therefore, the boundedness of $\lambda$ is not sufficient to guarantee that $\left\{g_{m, n}\right\}$ will form a Bessel sequence for $L^{2}(\mathbf{R})$.

The following theorem summarizes the connection between the lower or upper frame bounds and the function $\lambda$ for arbitrary $g \in L^{2}(\mathbf{R})$.

\subsection{Theorem.}

Let $g \in L^{2}(\mathbf{R})$.

(a) If there exists a constant $A>0$ such that

$$
\forall f \in L^{2}(\mathbf{R}), \quad A\|f\|_{2}^{2} \leq \sum_{m, n}\left|\left\langle f, g_{m, n}\right\rangle\right|^{2}
$$

then

$$
A \leq \sum_{n}|g(t-n)|^{2} \text { a.e. } \quad \text { and } \quad A \leq \sum_{m}|\hat{g}(\gamma-m)|^{2} \text { a.e. }
$$

(b) If $\left\{g_{m, n}\right\}$ is a Bessel sequence, i.e., there exists a constant $B>0$ such that

$$
\forall f \in L^{2}(\mathbf{R}), \quad \sum_{m, n}\left|\left\langle f, g_{m, n}\right\rangle\right|^{2} \leq B\|f\|_{2}^{2},
$$

then

$$
\sum_{n}|g(t-n)|^{2} \leq B \text { a.e. } \quad \text { and } \quad \sum_{m}|\hat{g}(\gamma-m)|^{2} \leq B \text { a.e. }
$$


Theorem 3.5 is a generalization of a result stated in [Dau90, $\S 2.3 .1 \mathrm{~B}]$ and proved in [HW89, Proposition 4.1.4]. The theorem as stated here was proved in [CS93, Theorem 2].

The remainder of this subsection is devoted to examining the converse of Theorem 3.5. First, the BLT implies that the converse of Theorem 3.5a fails.

\subsection{Proposition.}

There exists a function $g \in L^{2}(\mathbf{R})$ satisfying (3.5) but not (3.4).

Proof. Let $g(t)=e^{-\pi t^{2}}$. Then $g$ certainly satisfies (3.5) for some $A>0$. Moreover, $\left\{g_{m, n}\right\}$ is a Bessel sequence, that is, (3.6) holds [Dau90, Theorem 2.5], [HW89, Proposition 4.2.1], [Wal92, Theorem 3.1]. However, by the BLT, $\left\{g_{m, n}\right\}$ is not a frame. Therefore (3.4) cannot hold.

The converse of Theorem 3.5b is false as well.

\subsection{Proposition.}

There exists a function $g \in L^{2}(\mathbf{R})$ satisfying (3.7) but not (3.6).

Proof. If $g \in L^{2}(\mathbf{R})$ then, by the Plancherel formula for Fourier series,

$$
\sum_{n}|g(t+n)|^{2}=\int_{0}^{1}\left|\sum_{n} g(t+n) e^{2 \pi i n \omega}\right|^{2} d \omega=\int_{0}^{1}|Z g(t, \omega)|^{2} d \omega
$$

for almost every $t$. Since $|Z \hat{g}(\omega, t)|=|Z g(t,-\omega)|$, we also have

$$
\sum_{m}|\hat{g}(\omega+m)|^{2}=\int_{0}^{1}|Z g(t,-\omega)|^{2} d t
$$

for almost every $\omega$. Let $G(t, \omega)=|t-\omega|^{-1 / 4}$ for $(t, \omega) \in Q$. Then $\iint_{Q}|G(t, \omega)|^{2} d t d \omega=6$, so $G \in L^{2}(Q)$. Therefore $g=Z^{-1} G \in L^{2}(\mathbf{R})$, and $G=Z g$. Since

$$
\underset{t \in[0,1]}{\operatorname{ess} \sup } \int_{0}^{1}|G(t, \omega)|^{2} d \omega=\underset{\omega \in[0,1]}{\operatorname{esssup}} \int_{0}^{1}|G(t,-\omega)|^{2} d t=2 \sqrt{2}
$$

it follows from (3.8) and (3.9) that (3.7) holds. However, $Z g=G$ is unbounded on $Q$. Theorem 3.1c therefore implies that (3.6) does not hold.

\subsection{The Discrete Zak Transform}

Our main focus in this paper is on functions $f$ of a continuously defined independent variable $t$. However, practical applications often require discrete implementations. We therefore devote this 
section to discussing discrete analogues of Gabor systems and the Zak transform. Here the variable $t$ will be restricted to discrete values, that is, $t \in \mathbf{Z}$. This amounts to considering sequences $\{f(t)\}_{t \in \mathbf{Z}}$ in place of functions $f$. Our discussion is based on [Wal89b] and [Hei89]. However, as with the usual Zak transform, the discrete Zak transform has been independently considered by other authors, for example, [AGT92].

Let $g=\{g(t)\}_{t \in \mathbf{Z}}$ be a sequence in $\ell^{2}(\mathbf{Z})$, so that $\sum|g(t)|^{2}<\infty$. As with the usual Gabor systems, we define new sequences $g_{m b, n a}$ by translating and modulating $g$. We must restrict the values of $a$ and $b$ to those for which $a$ and $N=1 / b$ are integers. Then we define $g_{m b, n a}(t)=$ $e^{2 \pi i m b t} g(t-n a)$ for $t \in \mathbf{Z}$. The collection of sequences $\left\{g_{m b, n a}\right\}_{m \in \mathbf{Z}_{N}, n \in \mathbf{Z}}$, where $\mathbf{Z}_{N}=\{0,1, \ldots, N-$ $1\}$, is the discrete Gabor system generated by $g, a$, and $b$.

Many results for usual Gabor systems have analogues for discrete Gabor systems. For example, there is a discrete Zak transform that is especially useful for analyzing the case $a b=1$. Because the variable $t$ has been restricted to discrete values, it is more convenient to retain the given values of $a$ and $b$ rather than attempting to "dilate" $g$ in order to redefine $a$ and $b$ so that $a=b=1$. Therefore, we define the discrete Zak transform of a sequence $f \in \ell^{2}(\mathbf{Z})$ by

$$
Z_{d} f(t, \omega)=\sum_{k \in \mathbf{Z}} f(t+k a) e^{2 \pi i k \omega}, \quad(t, \omega) \in \mathbf{Z} \times \hat{\mathbf{R}} .
$$

Note that $Z_{d} f$ is quasiperiodic in the following sense:

$$
Z_{d} f(t+a, \omega)=e^{-2 \pi i \omega} Z_{d} f(t, \omega) \quad \text { and } \quad Z_{d} f(t, \omega+1)=Z_{d} f(t, \omega) .
$$

Therefore $Z_{d} f$ is completely determined by its values on the set $D=\mathbf{Z}_{a} \times[0,1)=\{0, \ldots, a-1\} \times$ $[0,1)$, which is a union of $a$ line segments in the plane. It is easy to show that $Z_{d}$ is a unitary mapping of $\ell^{2}(\mathbf{Z})$ onto $L^{2}(D)$. Note that if the sequence $\{f(t)\}_{t \in \mathbf{Z}}$ is obtained by sampling a continuous function $F \in L^{2}(\mathbf{R})$ at times $t / a$, that is, $f(t)=F(t / a)$ for $t \in \mathbf{Z}$, then $Z_{d} f(t, \omega)=Z F(t / a, \omega)$ for $(t, \omega) \in \mathbf{Z} \times \hat{\mathbf{R}}$.

Now we apply the discrete Zak transform to a discrete Gabor system satisfying $a b=1$. In this case $N=1 / b=a$. Therefore, given $g \in \ell^{2}(\mathbf{Z})$, we compute $Z_{d}\left(g_{m b, n a}\right)(t, \omega)=$ $e^{2 \pi i m t / a} e^{2 \pi i n \omega} Z_{d} g(t, \omega)$. As $\left\{a^{-1 / 2} e^{2 \pi i m t / a} e^{2 \pi i n \omega}\right\}_{m \in \mathbf{Z}_{a}, n \in \mathbf{Z}}$ is an orthonormal basis for $L^{2}(D)$, there is an exact analogue of Theorem 3.1 for discrete Gabor systems. In particular, $\left\{g_{m b, n a}\right\}_{m \in \mathbf{Z}_{a}, n \in \mathbf{Z}}$ is an exact frame for $\ell^{2}(\mathbf{Z})$ if and only if there exist constants $A, B>0$ such that $A \leq\left|Z_{d} g(t, \omega)\right|^{2} \leq$ $B$ for a.e. $(t, \omega) \in D$. However, the fact that $D$ is a disjoint union of line segments leads to the following conclusion: We can find continuous functions $G \in L^{2}(\mathbf{R})$ that do not generate exact Gabor frames for $L^{2}(\mathbf{R})$ but whose sampled versions $\{g(t)\}_{t \in \mathbf{Z}}$ with $g(t)=G(t / a)$ for $t \in \mathbf{Z}$ do generate exact Gabor frames for $\ell^{2}(\mathbf{Z})$. We can even construct these functions $G \in L^{2}(\mathbf{R})$ so that $\|t G(t)\|_{2}\|\gamma \hat{G}(\gamma)\|_{2}<\infty$ and $G \in W\left(C_{0}, \ell^{1}\right)$, so that both the BLT and the Amalgam BLT imply that $G$ cannot generate a Gabor frame for $L^{2}(\mathbf{R})$. The reason is clear: $Z_{d} g(t, \omega)=Z G(t / a, \omega)$ for $(t, \omega) \in D$. Therefore $Z_{d} g$ may be bounded above and below on $D$ even though the same is not true for $Z G$ on $Q=[0,1) \times[0,1)$.

3.8. Example. Let $G$ be the Gaussian function $G(t)=e^{-t^{2}}$. As $G \in \mathcal{S}(\mathbf{R})$, both the BLT and the Amalgam BLT imply that $G$ cannot generate an exact Gabor frame for $L^{2}(\mathbf{R})$. In fact, this can be verified directly: $Z G$ is continuous and has a single zero in $Q$, at the point $(t, \omega)=(1 / 2,1 / 2)$. Define $g(t)=G(t / a)$ for $t \in \mathbf{Z}$. Then $Z_{d} g(t, \omega)=Z G(t / a, \omega)$ for $(t, \omega) \in D$. However, if $a$ is odd then $(a / 2,1 / 2) \notin D$. Therefore $Z_{d} g$ is continuous and nonzero on each of the disjoint line segments making up $D$. As a consequence, $Z_{d} g$ is bounded both above and below on $D$, and therefore $\{g(t)\}_{t \in \mathbf{Z}}$ generates an exact Gabor frame for $\ell^{2}(\mathbf{Z})$. 


\section{Wilson Bases}

In [DJJ91], Daubechies, Jaffard and Journé proved a remarkable result which provides a means to circumvent the restrictions imposed by the BLT. Their construction, which follows [DJJ91], is a simplification of an earlier construction due to Wilson [Wil87], [SRWW87].

\subsection{Theorem.}

Suppose that $g \in L^{2}(\mathbf{R})$ is such that

(a) $\hat{g}$ is real-valued, and

(b) $\left\{g_{m, n / 2}\right\}$ is a tight frame for $L^{2}(\mathbf{R})$ with frame bound 2 .

Then the collection $\left\{\psi_{\ell, k}\right\}_{\ell \geq 0, k \in \mathbf{Z}}$ defined by

$$
\begin{array}{ll}
\psi_{0, k}(t)=g(t-k), & \ell=0, \\
\psi_{\ell, k}(t)=\sqrt{2} g(t-k / 2) \cos (2 \pi \ell t), & \ell \neq 0, k+\ell \text { even }, \\
\psi_{\ell, k}(t)=\sqrt{2} g(t-k / 2) \sin (2 \pi \ell t), & \ell \neq 0, k+\ell \text { odd },
\end{array}
$$

is an orthonormal basis for $L^{2}(\mathbf{R})$.

Note that $\psi_{0, k}=g_{0, k}$ and that $\psi_{\ell, k}=(1 / \sqrt{2})\left(g_{\ell, k / 2}+(-1)^{k+\ell} g_{-\ell, k / 2}\right)$ if $k+\ell$ is even and $\psi_{\ell, k}=(i / \sqrt{2})\left(g_{\ell, k / 2}+(-1)^{k+\ell} g_{-\ell, k / 2}\right)$ if $k+\ell$ is odd. Thus, $\left\{\psi_{\ell, k}\right\}$ consists of combinations of appropriately chosen elements from the Gabor system $\left\{g_{m, n / 2}\right\}$. Note that by the discussion in $\S 2.2$, this Gabor system is inexact: it is neither orthogonal nor a basis. However, there does exist a $g$ satisfying the hypotheses of Theorem 4.1 that has good smoothness and decay. In particular, we can construct such a $g$ so that $\|t g(t)\|_{2}\|\gamma \hat{g}(\gamma)\|_{2}<\infty$. It is therefore possible to construct Wilson orthonormal bases for $L^{2}(\mathbf{R})$ whose elements have good smoothness and decay. Moreover, these bases are constructed from linear combinations of translates and modulates of a single function. In this sense, Theorem 4.1 circumvents the restrictions of the BLT. An example of a function $g \in L^{2}(\mathbf{R})$ satisfying the hypotheses of Theorem 4.1 and such that both $g$ and $\hat{g}$ have exponential decay was constructed in [DJJ91].

Theorem 4.1 has been generalized by Auscher [Aus94].

\subsection{Theorem.}

Assume that $|g(t)| \leq C(1+|t|)^{-1-\epsilon}$ for some $\epsilon>0$. Then $\left\{\psi_{\ell, k}\right\}$ forms an orthonormal basis for $L^{2}(\mathbf{R})$ if and only if the following two conditions are satisfied.

$$
\begin{aligned}
& \forall k \in \mathbf{Z}, \quad \sum_{n} \overline{g\left(t-k-\frac{n}{2}\right)} g\left(t-\frac{n}{2}\right)=2 \delta_{0, k} \text { a.e., } \\
& \forall k \in \mathbf{Z}, \quad \sum_{n}(-1)^{n} \overline{g\left(t-k-\frac{n}{2}-\frac{1}{2}\right)} g\left(-t-\frac{n}{2}\right)=0 \text { a.e. }
\end{aligned}
$$

If $\hat{g}$ is real-valued then (4.3) is automatically satisfied (see [Aus94, $§ 5.2 .3]$ ). In this case, (4.2) is equivalent to the statement that $\left\{g_{m, n / 2}\right\}$ forms a tight frame for $L^{2}(\mathbf{R})$ with frame bound 2 [DJJ91], 
[Aus94]. Therefore, in order to construct a Wilson basis whose elements have good smoothness and decay, it suffices to construct a tight Gabor frame $\left\{g_{m, n / 2}\right\}$ such that $\hat{g}$ is real-valued and $g$ has both good smoothness and good decay. We shall construct examples of such functions.

Before doing this, we make some general remarks concerning Gabor frames of the form $\left\{g_{m, n / 2}\right\}$. Recall from $\S 2.1$ that the frame operator $S$ is a positive operator, and that $\left\{S^{-1 / 2}\left(g_{m, n / 2}\right)\right\}$ is a tight frame with bound 1 . We can show that this latter frame is also a Gabor frame, generated by the function $S^{-1 / 2} g$. Recall that $\|I-c S\|<1$, where $c=2 /(A+B)$ and $A, B$ are the frame bounds for $\left\{g_{m, n / 2}\right\}$. Then $S^{-1 / 2}$ can be realized as a norm-convergent power series in $I-c S$ :

$$
S^{-1 / 2}=c^{1 / 2} \sum_{k=0}^{\infty} a_{k}(I-c S)^{k}
$$

for some appropriate choice of real coefficients $a_{k}$. The frame operator $S$ commutes with each the operators $e_{m}$ and $\tau_{n / 2}$. Therefore each partial sum in (4.4) commutes with these operators, and therefore the limit $S^{-1 / 2}$ commutes with each $e_{m}$ and $\tau_{n / 2}$. Hence $S^{-1 / 2}\left(g_{m, n / 2}\right)=\left(S^{-1 / 2} g\right)_{m, n / 2}$, so the tight frame is a Gabor frame, generated by $S^{-1 / 2} g$.

The following result regarding general Gabor frames $\left\{g_{m b, n a}\right\}$ was proved in [Wal92].

\subsection{Theorem.}

Assume $\left\{g_{m b, n a}\right\}$ is a Gabor frame with frame operator $S$. If $\hat{g} \in W\left(L^{\infty}, \ell^{1}\right)$, then

$$
\forall f \in L^{2}(\mathbf{R}), \quad(S f)^{\wedge}=a^{-1} \sum_{j}\left(\tau_{j / a} \hat{f} \cdot \sum_{n} \tau_{n b}\left(\hat{g} \cdot \tau_{j / a} \overline{\hat{g}}\right)\right) .
$$

This says that, assuming good decay of $\hat{g},(S f)^{\wedge}$ can be realized solely in terms of translations

of $\hat{g}$ and $\hat{f}$. Suppose now that $\hat{g} \in W\left(C_{0}, \ell^{1}\right)$ and that $\hat{g}$ is real-valued. If $\hat{f}$ is also real-valued then, by $(4.5)$, so is $(S f)^{\wedge}$. Since the operator $S^{-1 / 2}$ can be realized as the norm-convergent sum (4.4) and since every partial sum is a linear combination of powers of $S$ with real-valued coefficients, $\left(S^{-1 / 2} f\right)^{\wedge}$ is also real-valued. Applying Theorem 4.1, we therefore obtain the following.

\subsection{Theorem.}

Suppose that $h \in L^{2}(\mathbf{R})$ is such that

(a) $\hat{h}$ is real-valued,

(b) $\hat{h} \in W\left(L^{\infty}, \ell^{1}\right)$, and

(c) $\left\{h_{m, n / 2}\right\}$ is a frame for $L^{2}(\mathbf{R})$.

Let $S$ be the frame operator for $\left\{h_{m, n / 2}\right\}$, and define $g=\sqrt{2} S^{-1 / 2} h$. Then the collection $\left\{\psi_{\ell, k}\right\}$ defined by (4.1) is an orthonormal basis for $L^{2}(\mathbf{R})$.

4.5. Example. We shall construct a function $g \in C_{c}^{\infty}(\mathbf{R})$ such that $\left\{\psi_{\ell, k}\right\}$ is an orthonormal basis for $L^{2}(\mathbf{R})$. A similar example appears in [DJJ91] and [Aus94]. Let $h \in C_{c}^{\infty}(\mathbf{R})$ be given such that 
(a) $\operatorname{supp}(h) \subseteq[-1 / 2,1 / 2]$,

(b) $h$ is even and real-valued (so $\hat{h}$ is real-valued as well), and

(c) there exist constants $A, B>0$ such that $A \leq \sum|h(t-n / 2)|^{2} \leq B$.

In this case, $\left\{h_{m, n / 2}\right\}$ forms a frame for $L^{2}(\mathbf{R})$ with frame bounds $A$ and $B$ [Dau90], [HW89]. The corresponding frame operator is $S f=f \cdot \lambda$, where $\lambda(t)=\sum|h(t-n / 2)|^{2}$. In addition, $S^{-1 / 2} f=f \cdot \lambda^{-1 / 2}$. Defining $g=\sqrt{2} h \cdot \lambda^{-1 / 2}$, we have that $\left\{g_{m, n / 2}\right\}$ is a tight frame with frame bound 2, and that $g \in C_{c}^{\infty}(\mathbf{R})$. Therefore, by Theorem 4.4, $\left\{\psi_{\ell, k}\right\}$ is an orthonormal basis for $L^{2}(\mathbf{R})$, and $\psi_{\ell, k} \in C_{c}^{\infty}(\mathbf{R})$ for each $\ell$ and $k$.

A similar construction can be performed on the Fourier transform side, resulting in a Wilson basis consisting of smooth, rapidly decaying functions which are bandlimited. Let $h$ be given such that $\hat{h} \in C_{c}^{\infty}(\hat{\mathbf{R}})$ and

(a) $\operatorname{supp}(\hat{h}) \subseteq[-1,1]$,

(b) $\hat{h}$ is real-valued, and

(c) there exist constants $A, B>0$ such that $A \leq \sum|\hat{h}(\gamma-m)|^{2} \leq B$.

Then $\left\{\hat{h}_{n / 2, m}\right\}$ forms a frame for $L^{2}(\mathbf{R})$ with frame bounds $A$ and $B$. Since $\left(h_{m, n / 2}\right)^{\wedge}=e^{\pi i m n} \hat{h}_{-n / 2, m}$, we conclude that $\left\{h_{m, n / 2}\right\}$ is a frame for $L^{2}(\mathbf{R})$. The corresponding frame operator is defined by $(S f)^{\wedge}=\hat{f} \cdot \Lambda$, where $\Lambda(\gamma)=\sum|\hat{h}(\gamma-m)|^{2}$, and $\left(S^{-1 / 2} f\right)^{\wedge}=\hat{f} \cdot \Lambda^{-1 / 2}$. If we define $g$ by $\hat{g}=\sqrt{2} \hat{h} \cdot \Lambda^{-1 / 2}$, then $\left\{g_{m, n / 2}\right\}$ is a tight frame with frame bound 2 for $L^{2}(\mathbf{R})$. Therefore, $\left\{\psi_{\ell, k}\right\}$ is an orthonormal basis for $L^{2}(\mathbf{R})$. Moreover, $\psi_{\ell, k} \in \mathcal{S}(\mathbf{R})$ and $\left(\psi_{\ell, k}\right)^{\wedge} \in C_{c}^{\infty}(\mathbf{R})$ for each $\ell$ and $k$.

It is possible to describe the frame operator $S$ for a Gabor frame $\left\{g_{m, n / 2}\right\}$ in terms of the Zak transform [Dau90], [Dau92], [HW89], cf. Theorem 3.1.

\subsection{Theorem.}

Given $g \in L^{2}(\mathbf{R})$. Then $\left\{g_{m, n / 2}\right\}$ is a frame for $L^{2}(\mathbf{R})$ with frame bounds $A, B$ if and only if

$$
0<A \leq|Z g(t, \omega)|^{2}+|Z g(t, \omega+1 / 2)|^{2} \leq B<\infty \text { a.e. }
$$

In this case, the frame operator $S$ is given by $Z(S f)=Z f \cdot M$, where $M(t, \omega)=|Z g(t, \omega)|^{2}+$ $|Z g(t, \omega+1 / 2)|^{2}$, and the operator $S^{-1 / 2}$ is given by $Z\left(S^{-1 / 2} f\right)=Z f \cdot M^{-1 / 2}$.

We shall use this result, along with the following theorem of Janssen [Jan82], to construct Wilson bases.

\subsection{Theorem.}

If $f \in \mathcal{S}(\mathbf{R})$, then $Z f \in C^{\infty}\left(\mathbf{R}^{2}\right)$ and is quasiperiodic. Conversely, if $F \in C^{\infty}\left(\mathbf{R}^{2}\right)$ is quasiperiodic, then there exists $f \in \mathcal{S}(\mathbf{R})$ such that $F=Z f$.

4.8. Example. We shall construct a function $g \in \mathcal{S}(\mathbf{R})$ such that $\left\{\psi_{\ell, k}\right\}$ is a Wilson basis for $L^{2}(\mathbf{R})$. Let $h(t)=e^{-\pi t^{2}}$. Then $h \in \mathcal{S}(\mathbf{R}), \hat{h}$ is real-valued, and $h$ satisfies (4.6) [Dau90], [Dau92]. 
By Theorem 4.7, $Z h \in C^{\infty}\left(\mathbf{R}^{2}\right)$ and is quasiperiodic. Moreover, (4.6) holds for some $A, B$, so $M(t, \omega)^{-1 / 2}=\left(|Z g(t, \omega)|^{2}+|Z g(t, \omega+1 / 2)|^{2}\right)^{-1 / 2}$ is in $C^{\infty}\left(\mathbf{R}^{2}\right)$, and is periodic with period 1 in both $t$ and $\omega$. Hence the function $G=Z h \cdot M^{-1 / 2}$ is in $C^{\infty}\left(\mathbf{R}^{2}\right)$ and is quasiperiodic. Therefore, by Theorem 4.7, $g=\sqrt{2} Z^{-1} G=\sqrt{2} S^{-1 / 2} h \in \mathcal{S}(\mathbf{R})$. Hence $\left\{\psi_{\ell, k}\right\}$ is an orthonormal basis for $L^{2}(\mathbf{R})$ with $\psi_{\ell, k} \in \mathcal{S}(\mathbf{R})$ for each $\ell$ and $k$.

A more general investigation of the continuity properties of a Gabor frame operator $S$ and the related operators $S^{-1}$ and $S^{-1 / 2}$ was conducted in [Wal92] and [Wal93]. The continuity of $S, S^{-1}$, and $S^{-1 / 2}$ on weighted spaces was examined in [Wal92]. In particular, for $s \geq 0$ let $w_{s}(t)=(1+|t|)^{s}$ or $w_{s}(t)=e^{s|t|}$, and define the weighted spaces

$$
\begin{aligned}
L_{s}^{p}(\mathbf{R}) & =\left\{f:\|f\|_{L_{s}^{p}}=\left\|f w_{s}\right\|_{p}<\infty\right\}, \\
\left(L_{s}^{p}\right)^{\wedge}(\mathbf{R}) & =\left\{f:\|f\|_{\left(L_{s}^{p}\right)^{\wedge}}=\left\|\hat{f} w_{s}\right\|_{p}<\infty\right\} .
\end{aligned}
$$

Membership of $f$ in $L_{s}^{p}$ quantifies the decay of $f$, and membership of $f$ in $\left(L_{s}^{p}\right)^{\wedge}$ quantifies the smoothness of $f$. Assume now that $g(t) w_{s}(t) \in W\left(L^{\infty}, \ell^{1}\right)$, and that $\hat{g}(\gamma) w_{s}(\gamma) \in W\left(L^{\infty}, \ell^{1}\right)$. Then for all sufficiently small $a, b>0$ (how small depends on $g$ and $s$ ), $S$ and $S^{1 / 2}$ are continuous bijections of $L_{s}^{p}(\mathbf{R})$ and $\left(L_{s}^{p}\right)^{\wedge}(\mathbf{R})$ onto themselves for all $1 \leq p<\infty$. In particular, for $a$ and $b$ small enough, $S^{-1 / 2} g$ has the same smoothness and decay properties as $g$, as measured by membership in the spaces $L_{s}^{p}$ and $\left(L_{s}^{p}\right)^{\wedge}$.

For the specific case of $a b=1 / 2$, it has been shown in [Wal93] that if $g \in \mathcal{S}(\mathbf{R})$ satisfies (4.6) then $S$ and $S^{1 / 2}$ are continuous bijections of the modulation spaces $M_{p, q}^{s}(\mathbf{R})$ onto themselves for $s \geq 0$ and $1 \leq p, q<\infty . M_{p, q}^{s}(\mathbf{R})$ is an example of a Wiener amalgam space, namely $M_{p, q}^{s}=W\left(\left(L_{s}^{q}\right)^{\wedge}, \ell^{p}\right)$ [Fei89], [Fei90]. Given a function $\theta \in C_{c}^{\infty}(\mathbf{R})$ satisfying $\sum \theta(t-n) \equiv 1, f \in M_{p, q}^{s}(\mathbf{R})$ if and only if $\sum\left\|f \cdot \tau_{n} \theta\right\|_{\left(L_{s}^{q}\right) \wedge}^{p}<\infty$. That is, $f \in M_{p, q}^{s}(\mathbf{R})$ is characterized by a local smoothness criterion $\left(f \cdot \tau_{n} \theta \in\left(L_{s}^{q}\right)^{\wedge}\right.$ for all $\left.n\right)$, and a global decay criterion $\left(\left\{\left\|f \cdot \tau_{n} \theta\right\|_{\left(L_{s}^{q}\right)}\right\} \in \ell^{p}\right)$.

The continuity and invertibility of $S$ and $S^{1 / 2}$ on $M_{p, q}^{s}(\mathbf{R})$ for $a b=1 / 2$ says that these operators preserve smoothness and decay as measured by membership in the spaces $M_{p, q}^{s}$. It has been shown that unconditional bases of Wilson-type exist for the modulation spaces [FGW92]. The continuity and invertibility of $S$ and $S^{1 / 2}$ on the modulation spaces has been used in [GW92] to construct sets of sampling and interpolation in the Bargmann-Fock spaces of entire functions.

A general construction that includes many examples of Wilson bases as well as wavelet bases are the local trigonometric bases of Coifman and Meyer [CM91]. Similar constructions were proposed in [Mal90] in a signal processing context, and in [Lae90] in the context of bases adapted to arbitrary decompositions of the frequency domain. For more details, and especially the connection with Wilson bases, see [Aus94] and [AWW92].

\section{Distributional Calculations and the Continuity of the Zak Transform}

We devote this section to investigating relationships between differentiation and the continuity of the Zak transform.

Distributional differentiation will be denoted by $\partial$ on $\mathbf{R}$ or $\partial_{j}$ on $\mathbf{R}^{d}$ (the $j$-th partial). Classical differentiation is denoted by $D$ on $\mathbf{R}$ and $D_{j}$ on $\mathbf{R}^{d}$. The Sobolev space $L_{1}^{2}\left(\mathbf{R}^{d}\right)$ is $\left\{f \in L^{2}\left(\mathbf{R}^{d}\right)\right.$ : $\left.\partial_{j} f \in L^{2}\left(\mathbf{R}^{d}\right), j=1, \ldots, d\right\}$. 
If $x=(t, \omega) \in \mathbf{R} \times \hat{\mathbf{R}}$ and $r>0$ then $Q(x ; r)=Q(t, \omega ; r)$ is the square centered at $x$ with side $r$,

$$
\begin{aligned}
Q(x ; r) & =[t-r / 2, t+r / 2) \times[\omega-r / 2, \omega+r / 2) \\
& =\{(u, \alpha) \in \mathbf{R} \times \hat{\mathbf{R}}: u \in[t-r / 2, t+r / 2), \alpha \in[\omega-r / 2, \omega+r / 2)\} .
\end{aligned}
$$

Thus the square $Q=[0,1) \times[0,1)$ defined in $\S 3.1$ is $Q=Q(1 / 2,1 / 2 ; 1)$.

The proof of the following result is routine, e.g., [Maz85, pp. 8-9], [Sch66, Theorem 2.III (pp. 53-54) and Theorem 2.V (pp. 57-59)].

\subsection{Theorem.}

a. Given $F \in L_{1}^{2}\left(\mathbf{R}^{d}\right)$, there is a function $\tilde{F}$ on $\mathbf{R}^{d}$ such that $\tilde{F}=F$ a.e. and $\tilde{F} \in A C_{\text {loc }}$ on almost all straight lines parallel to the coordinate axes. The classical gradient $\nabla \tilde{F}$ of $\tilde{F}$ exists a.e. on $\mathbf{R}^{d}$; and the distributional gradient of $F$ is the distribution corresponding to $\nabla \tilde{F}$.

b. Given $F \in L^{2}\left(\mathbf{R}^{d}\right)$, if there is a function $\tilde{F}$ on $\mathbf{R}^{d}$ such that $\tilde{F} \in A C_{\text {loc }}$ on almost all straight lines parallel to the coordinate axes, $\tilde{F}=F$ a.e., and $D_{j} \tilde{F} \in L^{2}\left(\mathbf{R}^{d}\right)$ for $j=1, \ldots, d$, then $\partial_{j} F$ is the distribution corresponding to $D_{j} \tilde{F}$ for $j=1, \ldots, d$, and, hence, $F \in L_{1}^{2}\left(\mathbf{R}^{d}\right)$.

$\mathbf{R}^{d}$ can be replaced by any open subset and $L_{1}^{2}$ can be replaced by any $L_{1}^{p}$ for $1 \leq p \leq \infty$.

\subsection{Theorem.}

Given $g \in L^{2}(\mathbf{R})$, then $\partial g \in L^{2}(\mathbf{R})$ if and only if $\gamma \hat{g}(\gamma) \in L^{2}(\hat{\mathbf{R}})$. In this case, $g \in A C_{\mathrm{loc}}$, $D g=\partial g$ a.e., and

$$
\partial g(t)=(2 \pi i \gamma \hat{g}(\gamma))^{\vee}(t) .
$$

Proof. a. Suppose $\partial g \in L^{2}(\mathbf{R})$. Since $L^{2}(\mathbf{R}) \subseteq \mathcal{S}^{\prime}(\mathbf{R})$ and $\gamma \hat{g}(\gamma) \in \mathcal{S}^{\prime}(\mathbf{R})$, we can compute

$$
\left\langle(\partial g)^{\wedge}, \varphi\right\rangle=-\langle g, \partial \breve{\varphi}\rangle=2 \pi i\left\langle g(t),(\gamma \varphi(\gamma))^{\vee}(t)\right\rangle=\langle 2 \pi i \gamma \hat{g}(\gamma), \varphi(\gamma)\rangle
$$

for each $\varphi \in \mathcal{S}(\hat{\mathbf{R}})$. Thus, $\gamma \hat{g}(\gamma) \in L^{2}(\hat{\mathbf{R}})$ and (5.1) holds. The same calculation, this time going right to left, proves that $\gamma \hat{g}(\gamma) \in L^{2}(\hat{\mathbf{R}})$ implies $\partial g \in L^{2}(\mathbf{R})$.

b. If $\partial g \in L^{2}(\mathbf{R})$, then

$$
h(t)=\int_{0}^{t} \partial g(u) d u
$$

is absolutely continuous on $[0,1]$. For each $k \in C_{c}^{\infty}(\mathbf{R})$ with $\operatorname{supp}(k) \subseteq(0,1)$, we have the following calculations:

$$
\int_{0}^{1} g(t) D k(t) d t=\langle g, \overline{D k}\rangle=-\langle\partial g, \bar{k}\rangle=-\int_{0}^{1} \partial g(t) k(t) d t
$$


and

$$
\int_{0}^{1} h(t) D k(t) d t=-\int_{0}^{1} D h(t) k(t) d t=-\int_{0}^{1} \partial g(t) k(t) d t .
$$

The first calculation is distributional while the second employs integration by parts and the fundamental theorem of calculus. Thus,

$$
\int_{0}^{1}(g(t)-h(t)) D k(t) d t=0
$$

for all such $k$, and hence, $g-h$ is constant a.e. on $[0,1]$. We conclude that $g \in A C_{\text {loc }}$ and $D g=\partial g$ a.e. A calculation such as this is involved in proving Theorem 5.1a.

5.3. Example. a. If $g, t g(t) \in L^{2}(\mathbf{R})$ and $\gamma \hat{g}(\gamma) \in L^{2}(\mathbf{R})$ then not only does $G=Z g \in L^{2}(Q)$, but also $\partial_{1} G, \partial_{2} G \in L_{\text {loc }}^{2}\left(\mathbf{R}^{2}\right)$. To see this, first note that

$$
\partial_{1} G(t, \omega)=\sum \partial g(t+k) e^{2 \pi i k \omega} \in L^{2}(Q),
$$

since $\partial g \in L^{2}(\mathbf{R})$ by Theorem 5.2. Next, we compute

$$
\partial_{2} G(t, \omega)=2 \pi i Z(t g(t))(t, \omega)-2 \pi i t Z g(t, \omega) \in L_{\mathrm{loc}}^{2}\left(\mathbf{R}^{2}\right),
$$

since $\operatorname{tg}(t) \in L^{2}(\mathbf{R})$.

b. If $F \in L_{\mathrm{loc}}^{1}\left(\mathbf{R}^{2}\right)$ and $\partial_{1} F, \partial_{2} F \in L_{\mathrm{loc}}^{2}\left(\mathbf{R}^{2}\right)$ then

$$
\begin{aligned}
& \frac{1}{|Q(t, \omega ; r)|} \iint_{Q(t, \omega ; r)}\left|F(u, \alpha)-F_{Q(u, \alpha ; r)}\right| d u d \alpha \\
& \leq 2\left(\iint_{Q(t, \omega ; 2 r)}\left|\partial_{1} F(u, \alpha)\right|^{2} d u d \alpha\right)^{1 / 2}+2\left(\iint_{Q(t, \omega ; 2 r)}\left|\partial_{2} F(u, \alpha)\right|^{2} d u d \alpha\right)^{1 / 2}
\end{aligned}
$$

where $Q(t, \omega ; r)$ designates the square $\{(u, \alpha):|u-t|,|\alpha-\omega| \leq r / 2\}$ centered at $(t, \omega)$ and

$$
F_{Q(t, \omega ; r)}=\frac{1}{|Q(t, \omega ; r)|} \iint_{Q(t, \omega ; r)} F(u, \alpha) d u d \alpha
$$

We mention this inequality because means related to $F_{Q}$ are used in the proof of Theorem 6.4. 
To verify (5.2), first write the left side as

$$
\begin{aligned}
& \frac{1}{r^{4}} \iint_{Q(t, \omega ; r)}\left|\iint_{Q(u, \alpha ; r)}(F(u, \alpha)-F(v, \beta)) d v d \beta\right| d u d \alpha \\
& \leq \frac{1}{r^{4}} \iint_{Q(t, \omega ; r)} \iint_{Q(u, \alpha ; r)}|F(u, \alpha)-F(v, \alpha)| d v d \beta d u d \alpha \\
&+\frac{1}{r^{4}} \iint_{Q(t, \omega ; r)} \iint_{Q(u, \alpha ; r)}|F(v, \alpha)-F(v, \beta)| d v d \beta d u d \alpha
\end{aligned}
$$

Next, by Theorem 5.1a, $F \in A C_{\text {loc }}$ on almost all straight lines parallel to the coordinate axes, and the classical and distributional gradients are equivalent. In particular, we can use the fundamental theorem of calculus as follows: for almost all $\alpha$,

$$
\forall u, v, \quad|F(u, \alpha)-F(v, \alpha)|=\left|\int_{u}^{v} \partial_{1} F(s, \alpha) d s\right|,
$$

and, for almost all $v$,

$$
\forall \alpha, \beta, \quad|F(v, \alpha)-F(v, \beta)|=\left|\int_{\alpha}^{\beta} \partial_{2} F(v, \theta) d \theta\right| .
$$

Thus, the right hand side of (5.3) is bounded by

$$
\begin{aligned}
I_{1}+I_{2}= & \frac{1}{r^{4}} \iint_{Q(t, \omega ; r)} \iint_{Q(u, \alpha ; r)}\left|\int_{u}^{v} \partial_{1} F(s, \alpha) d s\right| d v d \beta d u d \alpha \\
& +\frac{1}{r^{4}} \iint_{Q(t, \omega ; r)} \iint_{Q(u, \alpha ; r)}\left|\int_{\alpha}^{\beta} \partial_{2} F(v, \theta) d \theta\right| d v d \beta d u d \alpha .
\end{aligned}
$$

In $I_{1}, s$ is between $u$ and $v,|u-v| \leq r / 2$, and $|u-t| \leq r / 2$; hence,

$$
\begin{aligned}
I_{1} & \leq \frac{r^{3}}{r^{4}} \int_{t-r}^{t+r} \int_{\omega-r / 2}^{\omega+r / 2}\left|\partial_{1} F(s, \alpha)\right| d \alpha d s \\
& \leq \sqrt{2}\left(\iint_{Q(t, \omega ; 2 r)}\left|\partial_{1} F(s, \alpha)\right|^{2} d \alpha d s\right)^{1 / 2} .
\end{aligned}
$$

In $I_{2}, \theta$ is between $\alpha$ and $\beta,|\alpha-\beta| \leq r / 2$, and $|\alpha-\omega| \leq r / 2$; also, $|v-u| \leq r / 2$ and $|u-t| \leq r / 2$. Hence,

$$
\begin{aligned}
I_{2} & \leq \frac{r^{3}}{r^{4}} \int_{\omega-r}^{\omega+r} \int_{t-r}^{t+r}\left|\partial_{2} F(v, \theta)\right| d v d \theta \\
& \leq 2\left(\iint_{Q(t, \omega ; 2 r)}\left|\partial_{2} F(v, \theta)\right|^{2} d v d \theta\right)^{1 / 2} .
\end{aligned}
$$

(5.2) now follows. 
c. Let $g=\mathbf{1}_{[0,1)}$. Then $G(t, \omega)=Z g(t, \omega)=e^{-2 \pi i n \omega}$ for $t \in[n, n+1)$. Clearly, $g, t g(t) \in$ $L^{2}(\mathbf{R}), D g=0$ a.e., $\gamma \hat{g}(\gamma) \notin L^{2}(\hat{\mathbf{R}})$, and $\partial g=\delta-\delta_{1} \notin L^{2}(\mathbf{R})$. Also, $\left\{g_{m, n}\right\}$ is an orthonormal basis for $L^{2}(\mathbf{R})$.

This data is, of course, consistent with the BLT; and we mention it only because of part b, and especially (5.4), which is used in the proof of (5.2). In fact, $G(u, \alpha)-G(v, \alpha)$ has the form $e^{-2 \pi i n(u) \alpha}-e^{2 \pi i n(v) \alpha}$, whereas $D_{1} G=0$ on $\cup(n, n+1)$ and

$$
\partial_{1} G(s, \alpha)=\sum\left(\delta_{n}(s)-\delta_{n+1}(s)\right) \otimes e_{-n}(\alpha)
$$

and, consequently, (5.4) fails for $F=G$.

In this example, $D g$ exists for all but two points, and $D g \in L^{1}(\mathbf{R})$; whereas, $\gamma \hat{g}(\gamma) \notin L^{2}(\hat{\mathbf{R}})$, cf., Theorem 5.2. On the other hand, we know that if $D f$ exists for each point of $\mathbf{R}$ and $D f \in L_{\text {loc }}^{1}(\mathbf{R})$ then $f \in A C_{\text {loc }}$ by the Banach-Zarecki theorem; cf., for example, [Ben76, Section 4.6].

d. C. Fefferman and E. Stein (Acta Mathematica, 1972) implemented the sharp maximal function whose mid-point version is

$$
F^{\sharp}(t, \omega)=\sup _{r>0} \frac{1}{|Q(t, \omega ; r)|} \iint_{Q(t, \omega ; r)}\left|F(u, \alpha)-F_{Q(t, \omega ; r)}\right| d u d \alpha .
$$

This should be compared with the maximal version of the left-hand side of (5.2), viz.,

$$
F^{b}(t, \omega)=\sup _{r>0} \frac{1}{|Q(t, \omega ; r)|} \iint_{Q(t, \omega ; r)}\left|F(u, \alpha)-F_{Q(u, \alpha ; r)}\right| d u d \alpha .
$$

The sharp maximal function corresponding to $F$ is in $L^{\infty}\left(\mathbf{R}^{2}\right)$ if and only if $F \in \operatorname{BMO}\left(\mathbf{R}^{2}\right)$; and twice the Hardy-Littlewood maximal function bounds $F^{\sharp}$.

5.4. Remark. The BLT is reasonable not only from the point of view of modern physics, as demonstrated in [Bal81], [Low85], but also in the tradition of classical analysis. We list the following instances.

a. Given $g, t g(t) \in L^{2}(\mathbf{R})$ and $\gamma \hat{g}(\gamma) \in L^{2}(\hat{\mathbf{R}})$, and let $G=Z g$. Suppose

$$
\partial_{1}^{2} G, \partial_{2}^{2} G \in L_{\mathrm{loc}}^{2}\left(\mathbf{R}^{2}\right)
$$

(noting there are no mixed partials). Using Example 5.3a and (5.5) we have the hypotheses of that form of Sobolev's lemma that allows us to conclude that there is a continuous function $\tilde{G}$ on $\mathbf{R}^{2}$ for which $\tilde{G}=G$ a.e. By properties of the Zak transform, $\tilde{G}$ has zeros. Therefore, $\left\{g_{m, n}\right\}$ is not a frame. We have shown that the phenomenon exhibited by the BLT follows easily with the added hypothesis (5.5).

b. In the setting of $\mathbf{R}^{2}$, Krylov's theorem [Sch66, pp. 181-185] asserts that if a distribution $G$ has the properties $\partial_{1} G, \partial_{2} G \in L_{\text {loc }}^{2}\left(\mathbf{R}^{2}\right)$, then $G^{n} \in L_{\text {loc }}^{1}\left(\mathbf{R}^{2}\right)$ for all $n \geq 1$. Consequently, in the case $g, t g(t) \in L^{2}(\mathbf{R})$ and $\gamma \hat{g}(\gamma) \in L^{2}(\hat{\mathbf{R}})$, we conclude that $G=Z g$ almost has the upper bound property for $\left\{g_{m, n}\right\}$ to be a frame, that is, $G$ is almost an element of $L_{\text {loc }}^{\infty}\left(\mathbf{R}^{2}\right)$. This tantalizing suggestion contrary to the Balian-Low phenomenon is balanced by the following fact also based on Sobolev's work: If a distribution $G$ has the properties $\partial_{1} G, \partial_{2} G \in L_{\mathrm{loc}}^{2}\left(\mathbf{R}^{2}\right)$ then $G \in L_{\mathrm{loc}}^{1}\left(\mathbf{R}^{2}\right)$ 
satisfies an $L_{\text {loc }}^{p}$ Lipschitz condition [Sch66, pp. 185-188]. If $G$ were actually continuous we could use our argument from part a to verify the BLT.

In Remark 5.4a we gave a simple proof of the BLT in the case that additional smoothness, viz., (5.5), is assumed. This is a natural procedure to follow in implementing proofs depending on the continuity of the Zak transform. For example, whereas Remark 5.4a invoked Sobolev's lemma, the following results, Theorem 5.5 and Theorem 5.6, utilize a Wiener amalgam condition.

\subsection{Theorem.}

Given $g \in L^{1}(\mathbf{R})$, assume

$$
\partial g \in W\left(L^{\infty}, \ell^{1}\right)
$$

Then $\left\{g_{m, n}\right\}$ is not a frame.

Proof. Note that by (5.6), $g \in L_{1}^{1}(\mathbf{R})$. Therefore, by Theorem 5.1, $g$ is equal almost everywhere to a locally absolutely continuous function. Hence, we may assume that $g$ is continuous, and that the Fundamental Theorem of Calculus holds. To prove the theorem, it is sufficient to prove that $g \in W\left(C_{0}, \ell^{1}\right)$. We do this by verifying that $\sum \sup _{t \in[0,1]}|g(t+k)|<\infty$, that is,

$$
\sum_{k}\left|g\left(t_{k}+k\right)\right|<\infty
$$

for any sequence $\left\{t_{k}\right\} \subseteq[0,1]$.

Since $g \in L^{1}(\mathbf{R})$ we know that

$$
\sum_{k}|g(t+k)|<\infty \quad \text { a.e. on }[0,1] .
$$

Fixing one such $t$ in (5.8) we take the absolute value of the difference of the sums, (5.7) and (5.8). (To be proper, we consider finite sums.) This expression is bounded by

$$
\sum_{k}\left|g(t+k)-g\left(t_{k}+k\right)\right|
$$

which in turn is finite by (5.6). The result follows by routine manipulations.

As a consequence of Theorem 5.5, we obtain the following result.

\subsection{Theorem.}

Given $g, t g(t) \in L^{2}(\mathbf{R})$, and $\gamma \hat{g}(\gamma) \in L^{2}(\hat{\mathbf{R}})$, assume

$$
\sum_{n} n^{2}\left\|\partial g \cdot \mathbf{1}_{[n, n+1]}\right\|_{\infty}^{2}<\infty
$$

(in particular, $\partial g \in L^{\infty}(\mathbf{R})$ ). Then $\left\{g_{m, n}\right\}$ is not a frame. 


\section{Non-distributional Proof of the Balian-Low Theorem}

As stated in Theorem 1.1 (BLT), the decay and smoothness conditions, $g, \operatorname{tg}(t) \in L^{2}(\mathbf{R})$ and $\gamma \hat{g}(\gamma) \in L^{2}(\hat{\mathbf{R}})$, are sufficient to deny the frame property. In order to prove the BLT we shall assume both that $t g(t) \in L^{2}(\mathbf{R}), \gamma \hat{g}(\gamma) \in L^{2}(\hat{\mathbf{R}})$, and that $g$ generates a frame $\left\{g_{m, n}\right\}$ for $L^{2}(\mathbf{R})$. We shall obtain a contradiction by proving that $1 / Z g$ is unbounded and not that $Z g$ is continuous (which implies $1 / Z g$ is unbounded). All calculations are explicitly nondistributional, in contrast with the method of Coifman, Semmes, and Daubechies [Dau90] in which calculations are implicitly distributional.

Our first step in proving the BLT for $g \in L^{2}(\mathbf{R})$ is to bound the variation of $G=Z g$ over small squares $Q(x ; r) \subseteq \mathbf{R} \times \hat{\mathbf{R}}$. This result, Theorem 6.4, is reminiscent of Hardy and Littlewood's (1928)

characterization of one-dimensional bounded variation in terms of the $L^{1}$-norm (cf., for example [Ben76, Theorem 4.4]).

\subsection{Lemma.}

Let $f, t f(t)$, and $\gamma \hat{f}(\gamma)$ be square-integrable, set $F=Z f$, and let $\varepsilon \in \mathbf{R}$ and $x_{0} \in[-3 / 2,3 / 2] \times$ $\hat{\mathbf{R}}$ be given. Then

$$
\begin{gathered}
\left(\iint_{Q\left(x_{0} ; 1\right)}|F(t-\varepsilon, \omega)-F(t, \omega)|^{2} d t d \omega\right)^{1 / 2} \leq 2 \pi|\varepsilon|\left(\int|\gamma \hat{f}(\gamma)|^{2} d \gamma\right)^{1 / 2} \\
\left(\iint_{Q\left(x_{0} ; 1\right)}|F(t, \omega-\varepsilon)-F(t, \omega)|^{2} d t d \omega\right)^{1 / 2} \\
\leq 2 \pi|\varepsilon|\left[\left(\int|t f(t)|^{2} d t\right)^{1 / 2}+2\left(\int|f(t)|^{2} d t\right)^{1 / 2}\right] .
\end{gathered}
$$

Proof. To prove (6.1) note that

$$
F(t-\varepsilon, \omega)=\sum_{k} f(t-\varepsilon+k) e^{2 \pi i k \omega}=Z\left(\tau_{\varepsilon} f\right)(t, \omega) .
$$

Thus, by the unitarity of $Z$ and the Plancherel theorem,

$$
\begin{aligned}
\left(\iint_{Q\left(x_{0} ; 1\right)}|F(t-\varepsilon, \omega)-F(t, \omega)|^{2} d t d \omega\right)^{1 / 2} & =\left(\iint_{Q\left(x_{0} ; 1\right)}\left|Z\left(\tau_{\varepsilon} f-f\right)(t, \omega)\right|^{2} d t d \omega\right)^{1 / 2} \\
& =\left(\int\left|\tau_{\varepsilon} f(t)-f(t)\right|^{2} d t\right)^{1 / 2} \\
& =\left(\int\left|e^{-2 \pi i \varepsilon \gamma}-1\right|^{2}|\hat{f}(\gamma)|^{2} d \gamma\right)^{1 / 2} \\
& =\left(\int|2 \sin (\pi \varepsilon \gamma)|^{2}|\hat{f}(\gamma)|^{2} d \gamma\right)^{1 / 2} \\
& \leq 2 \pi|\varepsilon|\left(\int|\gamma \hat{f}(\gamma)|^{2} d \gamma\right)^{1 / 2}
\end{aligned}
$$


To prove (6.2), note that

$$
\begin{aligned}
F(t, \omega-\varepsilon) & =\sum_{k} f(t+k) e^{2 \pi i k(\omega-\varepsilon)} \\
& =e^{2 \pi i \varepsilon t} \sum_{k} e^{-2 \pi i \varepsilon(t+k)} f(t+k) e^{2 \pi i k \omega} \\
& =e^{2 \pi i \varepsilon t} Z\left(e_{-\varepsilon} f\right)(t, \omega) .
\end{aligned}
$$

By the unitarity of $Z$ and the Plancherel theorem we therefore have

$$
\begin{aligned}
&\left(\iint_{Q\left(x_{0} ; 1\right)}|F(t, \omega-\varepsilon)-F(t, \omega)|^{2} d t d \omega\right)^{1 / 2} \\
&=\left(\iint_{Q\left(x_{0} ; 1\right)}\left|e^{2 \pi i \varepsilon t} Z\left(e_{-\varepsilon} f\right)(t, \omega)-Z f(t, \omega)\right|^{2} d t d \omega\right)^{1 / 2} \\
& \leq\left(\iint_{Q\left(x_{0} ; 1\right)}\left|Z\left(e_{-\varepsilon} f-f\right)(t, \omega)\right|^{2} d t d \omega\right)^{1 / 2} \\
&+\left(\iint_{Q\left(x_{0} ; 1\right)}\left|e^{2 \pi i \varepsilon t}-1\right|^{2}|Z f(t, \omega)|^{2} d t d \omega\right)^{1 / 2} \\
&=\left(\int|2 \sin \pi \varepsilon t|^{2}|f(t)|^{2} d t\right)^{1 / 2}+\left(\iint_{Q\left(x_{0} ; 1\right)}|2 \sin \pi \varepsilon t|^{2}|Z f(t, \omega)|^{2} d t d \omega\right)^{1 / 2} \\
& \leq 2 \pi|\varepsilon|\left[\left(\int|t f(t)|^{2} d t\right)^{1 / 2}+2\left(\int|f(t)|^{2} d t\right)^{1 / 2}\right] \cdot \square
\end{aligned}
$$

Next we shall prove a result (Lemma 6.3) similar to the above, obtaining a bound on the oscillation of $F$ over a square of side $r<1$. First, we fix some notation and prove some technicalities in the following lemma.

\subsection{Lemma.}

Let $f \in L^{2}(\mathbf{R})$, and let $s \in \mathbf{R}$ and $r>0$ be given. Let $E(s, r)$ denote the set

$$
E(s, r)=\bigcup_{n \in \mathbf{Z}}\left[s-\frac{r}{2}+n, s+\frac{r}{2}+n\right]
$$

Then

(a) $Z f(t, \omega)=e^{-2 \pi i t \omega} Z \hat{f}(-\omega, t)=e^{-2 \pi i t \omega} Z \check{f}(\omega,-t)$,

(b) $Z f(t, \omega) \cdot \mathbf{1}_{E(s, r)}(t)=Z\left(f \cdot \mathbf{1}_{E(s, r)}\right)(t, \omega)$ for all $(t, \omega) \in \mathbf{R} \times \hat{\mathbf{R}}$,

(c) $\lim _{r \rightarrow 0}\left\|f \cdot \mathbf{1}_{E(s, r)}\right\|_{2}=0$ uniformly in $s$.

Proof. To prove a, use the Poisson Summation Formula for $f \in \mathcal{S}(\mathbf{R})$, then use the density of $\mathcal{S}(\mathbf{R})$ in $L^{2}(\mathbf{R})$ to complete the proof (cf. [Jan82], [Hei90]). Statement b follows immediately from 
the definition of the Zak transform and the fact that $\mathbf{1}_{E(s, r)}$ is 1-periodic. To prove c, first fix any $\varepsilon>0$. Since $|f|^{2}$ is an integrable function, we can find an $N>0$ such that $\int_{|t|>N}|f(t)|^{2} d t \leq \varepsilon / 2$, and a $\delta>0$ such that $\int_{A}|f(t)|^{2} d t \leq \varepsilon / 2$ whenever $|A| \leq \delta$. Now, given any $s \in \mathbf{R}$, the number of $n \in \mathbf{Z}$ such that $[s-r / 2+n, s+r / 2+n] \cap[-N, N] \neq \emptyset$ is at most $2 N+r+1$, which is independent of $s$. Therefore, $|E(s, r) \cap[-N, N]| \leq(2 N+2) r$ for all $s$ when $r \leq 1$. Letting $r_{0}=\delta /(2 N+2)$, we therefore have

$$
\left\|f \cdot \mathbf{1}_{E(s, r)}\right\|_{2}^{2} \leq \int_{|t|>N}|f(t)|^{2} d t+\int_{E(s, r) \cap[-N, N]}|f(t)|^{2} d t \leq \varepsilon
$$

for all $s$ and all $r \leq r_{0}$.

\subsection{Lemma.}

Let $f, t f(t)$, and $\gamma \hat{f}(\gamma)$ be square integrable; set $F=Z f$; and let $\varepsilon \in \mathbf{R},\left(t_{0}, \omega_{0}\right) \in[-3 / 2,3 / 2] \times$ $\hat{\mathbf{R}}$, and $0<r<1$ be given. Then

$$
\begin{gathered}
\left(\iint_{Q\left(t_{0}, \omega_{0} ; r\right)}|F(t-\varepsilon, \omega)-F(t, \omega)|^{2} d t d \omega\right)^{1 / 2} \leq 2 \pi|\varepsilon|\left\|\gamma \hat{f}(\gamma) \cdot \mathbf{1}_{E\left(1-\omega_{0}, r\right)}(\gamma)\right\|_{2} \\
\left(\iint_{Q\left(t_{0}, \omega_{0} ; r\right)}|F(t, \omega-\varepsilon)-F(t, \omega)|^{2} d t d \omega\right)^{1 / 2} \\
\leq 2 \pi|\varepsilon|\left(\left\|t f(t) \cdot \mathbf{1}_{E\left(t_{0}, r\right)}(t)\right\|_{2}+2\left\|f \cdot \mathbf{1}_{E\left(t_{0}, r\right)}\right\|_{2}\right) .
\end{gathered}
$$

Proof. Using arguments similar to those in the proof of Lemma 6.1, and parts a and b of Lemma 6.2, we have

$$
\begin{aligned}
\left(\iint_{Q\left(t_{0}, \omega_{0} ; r\right)}|F(t-\varepsilon, \omega)-F(t, \omega)|^{2} d t d \omega\right)^{1 / 2} & \left(\iint_{Q\left(t_{0}, \omega_{0} ; r\right)}\left|Z\left(\tau_{\varepsilon} f-f\right)(t, \omega)\right|^{2} d t d \omega\right)^{1 / 2} \\
= & \left(\iint_{Q\left(t_{0}, \omega_{0} ; r\right)}\left|Z\left(e_{-\varepsilon} \hat{f}-\hat{f}\right)(-\omega, t)\right|^{2} d t d \omega\right)^{1 / 2} \\
= & \left(\iint_{Q\left(t_{0},-\omega_{0} ; r\right)}\left|Z\left(e_{-\varepsilon} \hat{f}-\hat{f}\right)(\omega, t)\right|^{2} d \omega d t\right)^{1 / 2} \\
= & \left(\iint_{Q\left(t_{0}, 1-\omega_{0} ; r\right)}\left|Z\left(e_{-\varepsilon} \hat{f}-\hat{f}\right)(\omega, t)\right|^{2} d \omega d t\right)^{1 / 2} \\
= & \left(\iint_{Q\left(t_{0}, 1-\omega_{0} ; r\right)}\left|Z\left(e_{-\varepsilon} \hat{f}-\hat{f}\right)(\omega, t) \cdot \mathbf{1}_{E\left(1-\omega_{0}, r\right)}(\omega)\right|^{2} d \omega d t\right)^{1 / 2}
\end{aligned}
$$




$$
\begin{aligned}
& \leq\left(\iint_{Q\left(t_{0}, 1-\omega_{0} ; 1\right)}\left|Z\left(\left(e_{-\varepsilon} \hat{f}-\hat{f}\right) \cdot \mathbf{1}_{E\left(1-\omega_{0}, r\right)}\right)(\omega, t)\right|^{2} d \omega d t\right)^{1 / 2} \\
& =\left(\int_{E\left(1-\omega_{0}, r\right)}\left|e_{-\varepsilon} \hat{f}(\gamma)-\hat{f}(\gamma)\right|^{2} d \gamma\right)^{1 / 2} \\
& \leq 2 \pi|\varepsilon|\left\|\gamma \hat{f}(\gamma) \cdot \mathbf{1}_{E\left(1-\omega_{0}, r\right)}(\gamma)\right\|_{2},
\end{aligned}
$$

from which (6.3) follows.

For (6.4), we have that

$$
\begin{aligned}
&\left(\iint_{Q\left(t_{0}, \omega_{0} ; r\right)}|F(t, \omega-\varepsilon)-F(t, \omega)|^{2} d t d \omega\right)^{1 / 2} \\
& \leq\left(\iint_{Q\left(t_{0}, \omega_{0} ; r\right)}\left|Z\left(e_{-\varepsilon} f-f\right)(t, \omega)\right|^{2} d t d \omega\right)^{1 / 2} \\
&+\left(\iint_{Q\left(t_{0}, \omega_{0} ; r\right)}\left|e^{2 \pi i \varepsilon t}-1\right|^{2}|Z f(t, \omega)|^{2} d t d \omega\right)^{1 / 2} \\
& \leq\left(\iint_{Q\left(t_{0}, \omega_{0} ; r\right)}\left|Z\left(e_{-\varepsilon} f-f\right)(t, \omega) \cdot \mathbf{1}_{E\left(t_{0}, r\right)}(t)\right|^{2} d t d \omega\right)^{1 / 2} \\
&+2 \pi|\varepsilon|\left(\iint_{Q\left(t_{0}, \omega_{0} ; r\right)}\left|t Z f(t, \omega) \cdot \mathbf{1}_{E\left(t_{0}, r\right)}(t)\right|^{2} d t d \omega\right)^{1 / 2} \\
& \leq\left(\iint_{Q\left(t_{0}, \omega_{0} ; 1\right)}\left|Z\left(\left(e_{-\varepsilon} f-f\right) \cdot \mathbf{1}_{E\left(t_{0}, r\right)}\right)(t, \omega)\right|^{2} d t d \omega\right)^{1 / 2} \\
&+4 \pi|\varepsilon|\left(\iint_{Q\left(t_{0}, \omega_{0} ; r\right)}\left|Z\left(f \cdot \mathbf{1}_{E\left(t_{0}, r\right)}\right)(t, \omega)\right|^{2} d t d \omega\right)^{1 / 2} \\
&=\left(\int_{E\left(t_{0}, r\right)}\left|e_{-\varepsilon} f(t)-f(t)\right|^{2} d t\right)^{1 / 2}+4 \pi|\varepsilon|\left(\int_{E\left(t_{0}, r\right)}|f(t)|^{2} d t\right)^{1 / 2} .
\end{aligned}
$$

This last term is bounded by the right side of (6.4).

\subsection{Theorem.}

Let $f, \varepsilon, r$, and $\left(t_{0}, \omega_{0}\right)$ be as in Lemma 6.3 and in addition fix $0<c<1$. Then there are functions $C_{1}(r)$ and $C_{2}(r)$, depending on $f$ alone, such that $\lim _{r \rightarrow 0} C_{i}(r)=0$ for $i=1,2$ and

$$
\begin{aligned}
& \iint_{Q\left(t_{0}, \omega_{0} ; c r\right)}|F(t-\varepsilon, \omega)-F(t, \omega)| d t d \omega \leq c r|\varepsilon| C_{1}(r), \\
& \iint_{Q\left(t_{0}, \omega_{0} ; c r\right)}|F(t, \omega-\varepsilon)-F(t, \omega)| d t d \omega \leq c r|\varepsilon| C_{2}(r) .
\end{aligned}
$$


Proof. This result follows from Lemma 6.3 and a straightforward application of Hölder's inequality, where

$$
C_{1}(r)=2 \pi \sup _{\omega \in \hat{\mathbf{R}}}\left\|\gamma \hat{f}(\gamma) \cdot \mathbf{1}_{E(\omega, r)}(\gamma)\right\|_{2}
$$

and

$$
C_{2}(r)=2 \pi \sup _{t \in \mathbf{R}}\left(\left\|t f(t) \cdot \mathbf{1}_{E(t, r)}(t)\right\|_{2}+2\left\|f \cdot \mathbf{1}_{E(t, r)}\right\|_{2}\right) .
$$

The convergence to 0 of $C_{i}$ for $i=1,2$ follows from Lemma 6.2c.

The next step in our proof of the BLT is to introduce a continuous approximation $G_{r}$ of $G=Z g$. This idea is due to Coifman and Semmes, e.g., [Dau90], cf., Example 5.3b. It turns out that $G_{r}$ satisfies enough of a quasiperiodicity condition to allow a proof of the BLT similar to those which proceed by proving the continuity of the Zak transform.

Let $\rho=\mathbf{1}_{Q}$ and, for $r>0$, let $\rho_{r}(t, \omega)=r^{-2} \rho(t / r, \omega / r)$. Then $\left\{\rho_{r}\right\}$ is an approximate identity on $\mathbf{R} \times \hat{\mathbf{R}}$ as $r \rightarrow 0$. We define the mean-value function

$$
G_{r}(t, \omega)=\iint_{Q} G(t-u, \omega-\alpha) \rho_{r}(u, \alpha) d u d \alpha
$$

cf. the role of the mean value $F_{Q}$ defined after (6.2). Some basic properties of the function $G_{r}$ are the following, for example, [Dau90].

\subsection{Theorem.}

Let $g \in L^{2}(\mathbf{R})$ generate the frame $\left\{g_{m, n}\right\}$ with frame bounds $A$ and $B$, and let $G=Z g$. Then

(a) $\left|G_{r}\left(t_{1}, \omega_{1}\right)-G_{r}\left(t_{2}, \omega_{2}\right)\right| \leq 2 r^{-1} B^{1 / 2}\left(\left|t_{1}-t_{2}\right|+\left|\omega_{1}-\omega_{2}\right|\right)$,

(b) $\sup _{(t, \omega) \in Q}\left|G_{r}(t, \omega)\right| \leq B^{1 / 2}$,

(c) $G_{r}(t, \omega+1)=G_{r}(t, \omega)$,

(d) $G_{r}(t+1, \omega)=e^{-2 \pi i \omega} G_{r}(t, \omega)+\psi_{r}(t, \omega)$, where $\sup _{(t, \omega) \in Q}\left|\psi_{r}(t, \omega)\right| \leq \pi r B^{1 / 2}$,

(e) for fixed $(u, \alpha),(t, \omega) \in \mathbf{R} \times \hat{\mathbf{R}}$,

$$
\left|G(u, \alpha)-G_{r}(u, \alpha)\right| \geq A^{1 / 2}-2 r^{-1} B^{1 / 2}(|t-u|+|\omega-\alpha|)-\left|G_{r}(t, \omega)\right|
$$

and, in particular, for fixed $(t, \omega) \in Q$ and $c<1$,

$$
\inf _{(u, \alpha) \in Q(t, \omega ; c r)}\left|G(u, \alpha)-G_{r}(u, \alpha)\right| \geq A^{1 / 2}-2 c B^{1 / 2}-\left|G_{r}(t, \omega)\right| .
$$


Proof. a. Note that

$$
\begin{aligned}
\left|G_{r}\left(t_{1}, \omega_{1}\right)-G_{r}\left(t_{2}, \omega_{2}\right)\right| & =\frac{1}{r^{2}}\left|\iint_{Q\left(x_{1} ; r\right)} G(u, \alpha) d u d \alpha-\iint_{Q\left(x_{2} ; r\right)} G(u, \alpha) d u d \alpha\right| \\
& =\frac{1}{r^{2}}\left|\iint_{Q\left(x_{1} ; r\right) \Delta Q\left(x_{2} ; r\right)} G(u, \alpha) d u d \alpha\right| \\
& \leq \frac{B^{1 / 2}}{r^{2}}\left|Q\left(x_{1} ; r\right) \Delta Q\left(x_{2} ; r\right)\right|,
\end{aligned}
$$

where $\Delta$ is the symmetric difference operator, $x_{j}=\left(t_{j}-\frac{r}{2}, \omega_{j}-\frac{r}{2}\right)$ for $j=1,2$, and we have used the fact that $|G(u, \alpha)| \leq B^{1 / 2}$ a.e. (cf. Theorem 3.1d).

It remains to show that

$$
\left|Q\left(x_{1} ; r\right) \Delta Q\left(x_{2} ; r\right)\right| \leq 2 r\left(\left|t_{1}-t_{2}\right|+\left|\omega_{1}-\omega_{2}\right|\right) .
$$

This is certainly true if $Q\left(x_{1} ; r\right)$ and $Q\left(x_{2} ; r\right)$ are disjoint, so assume that these two squares overlap. Without loss of generality, we may assume that $t_{1} \leq t_{2}$ and $\omega_{1} \leq \omega_{2}$. Then

$$
Q\left(x_{1} ; r\right) \backslash Q\left(x_{2} ; r\right)=\left(t_{1}-r, t_{2}-r\right) \times\left(\omega_{1}-r, \omega_{1}\right) \cup\left(t_{1}-r, t_{1}\right) \times\left(\omega_{1}-r, \omega_{2}-r\right),
$$

so

$$
\left|Q\left(x_{1} ; r\right) \backslash Q\left(x_{2} ; r\right)\right| \leq r\left|t_{1}-t_{2}\right|+r\left|\omega_{1}-\omega_{2}\right| .
$$

The same inequality holds for $\left|Q\left(x_{2} ; r\right) \backslash Q\left(x_{1} ; r\right)\right|$, so part a follows upon noting that $Q\left(x_{1} ; r\right) \Delta Q\left(x_{2} ; r\right)=$ $Q\left(x_{1} ; r\right) \backslash Q\left(x_{2} ; r\right) \cup Q\left(x_{2} ; r\right) \backslash Q\left(x_{1} ; r\right)$.

b. Fix $(t, \omega) \in Q$, and note that by Theorem $3.1 \mathrm{~d}$,

$$
\begin{aligned}
\left|G_{r}(t, \omega)\right| & =\left|\iint_{Q} G(t-u, \omega-\alpha) \rho_{r}(u, \alpha) d u d \alpha\right| \\
& \leq B^{1 / 2} \iint_{Q} \rho_{r}(u, \alpha) d u d \alpha \\
& =B^{1 / 2} .
\end{aligned}
$$

c. Part c follows immediately from the fact that $G$ is periodic in the second variable.

d. Note that

$$
\begin{aligned}
G_{r}(t+1, \omega) & =\iint_{Q} G(t+1-u, \omega-\alpha) \rho_{r}(u, \alpha) d u d \alpha \\
& =\iint_{Q} e^{-2 \pi i(\omega-\alpha)} G(t-u, \omega-\alpha) \rho_{r}(u, \alpha) d u d \alpha \\
& =e^{-2 \pi i \omega} \iint_{Q} G(t-u, \omega-\alpha) \rho_{r}(u, \alpha) d u d \alpha+\psi_{r}(t, \omega)
\end{aligned}
$$


where

$$
\psi_{r}(t, \omega)=e^{-2 \pi i \omega} \iint_{Q}\left(e^{2 \pi i \alpha}-1\right) G(t-u, \omega-\alpha) \rho_{r}(u, \alpha) d u d \alpha
$$

We estimate

$$
\begin{aligned}
\left|\psi_{r}(t, \omega)\right| & \leq \iint_{Q}\left|e^{2 \pi i \alpha}-1\right||G(t-u, \omega-\alpha)| \rho_{r}(u, \alpha) d u d \alpha \\
& \leq B^{1 / 2} \iint_{Q}|2 \pi \alpha| \rho_{r}(u, \alpha) d u d \alpha \\
& \leq B^{1 / 2} \pi r
\end{aligned}
$$

e. Observe from a that

$$
\begin{aligned}
\left|G(u, \alpha)-G_{r}(u, \alpha)\right| & \geq|G(u, \alpha)|-\left|G_{r}(u, \alpha)-G_{r}(t, \omega)\right|-\left|G_{r}(t, \omega)\right| \\
& \geq A^{1 / 2}-\frac{2}{r} B^{1 / 2}(|t-u|+|\omega-\alpha|)-\left|G_{r}(t, \omega)\right| .
\end{aligned}
$$

Since $(u, \alpha) \in Q(t, \omega ; c r)$ implies that $|t-u|$ and $|\omega-\alpha| \leq c r / 2$, it follows that

$$
\inf _{(u, \alpha) \in Q(t, \omega ; c r)}\left|G(u, \alpha)-G_{r}(u, \alpha)\right| \geq A^{1 / 2}-2 c B^{1 / 2}-\left|G_{r}(t, \omega)\right| .
$$

\subsection{Lemma.}

Suppose that $g, \operatorname{tg}(t)$, and $\gamma \hat{g}(\gamma)$ are square-integrable. Then for any $(t, \omega) \in Q, c<1$, and $r<1$, we have that

$$
\iint_{Q(t, \omega ; c r)}\left|G(u, \alpha)-G_{r}(u, \alpha)\right| d u d \alpha \leq c r^{2} C(r),
$$

where $C(r)$ does not depend on $(t, \omega)$ and $\lim _{r \rightarrow 0} C(r)=0$.

Proof.

$$
\begin{aligned}
& \iint_{Q(t, \omega ; c r)}\left|G(u, \alpha)-G_{r}(u, \alpha)\right| d u d \alpha \\
& \leq \iint_{Q(t, \omega ; c r)} \iint_{Q}\left|\rho_{r}(v, \beta)\right||G(u, \alpha)-G(u-v, \alpha-\beta)| d v d \beta d u d \alpha \\
&= \iint_{Q}\left|\rho_{r}(v, \beta)\right| \iint_{Q(t, \omega ; c r)}|G(u, \alpha)-G(u-v, \alpha-\beta)| d u d \alpha d v d \beta \\
& \leq \iint_{Q}\left|\rho_{r}(v, \beta)\right| \iint_{Q(t, \omega ; c r)}|G(u, \alpha)-G(u-v, \alpha)| d u d \alpha d v d \beta \\
&+\iint_{Q}\left|\rho_{r}(v, \beta)\right| \iint_{Q(t-v, \omega ; c r)}|G(s, \alpha)-G(s, \alpha-\beta)| d s d \alpha d v d \beta
\end{aligned}
$$


where we have simply applied the triangle inequality. Since $\rho_{r}$ is supported in the square $Q(r / 2, r / 2 ; r)$ and since $r<1$, we have that $t-v \in[-1 / 2,1]$. Thus, by Theorem 6.4 , the above quantity is bounded by

$$
c r^{2}\left(C_{1}(r)+C_{2}(r)\right) .
$$

Putting $C(r)=C_{1}(r)+C_{2}(r)$ completes the proof.

Proof of Theorem 1.1 (BLT). First, we claim that $\left|G_{r}(t, \omega)\right| \geq A^{1 / 2} / 2$ for all $(t, \omega) \in Q$ and all $r$ sufficiently small. To see this, note that by Theorem $6.5 \mathrm{e}$ and Lemma 6.6 ,

$$
c r^{2} C(r) \geq \iint_{Q(t, \omega ; c r)}\left|G(u, \alpha)-G_{r}(u, \alpha)\right| d u d \alpha \geq c^{2} r^{2}\left(A^{1 / 2}-2 c B^{1 / 2}-\left|G_{r}(t, \omega)\right|\right) .
$$

Fixing $c$ so small that $A^{1 / 2}-2 c B^{1 / 2}>A^{1 / 2} / 2$, we have that

$$
\left|G_{r}(t, \omega)\right| \geq A^{1 / 2}-2 c B^{1 / 2}-\frac{C(r)}{c}
$$

Since $C(r) \rightarrow 0$ as $r \rightarrow 0$, independently of $(t, \omega)$, the claim is proved. such that

Thus, for each $r>0$, there exists a continuous, real-valued function $\varphi_{r}$ on $\bar{Q}=[0,1] \times[0,1]$

$$
G_{r}(t, \omega)=\left|G_{r}(t, \omega)\right| e^{i \varphi_{r}(t, \omega)}
$$

(for example, [RR55, pp. 377-385]). Then, as in the proof that continuous quasiperiodic functions have zeros, we have that for $t \in[0,1]$,

$$
\varphi_{r}(t, 1)=\varphi_{r}(t, 0)+2 \pi k_{r}
$$

for some integer $k_{r}$ independent of $t$ (for example, [HW89]).

Now let us consider the function

$$
\delta_{r}(t, \omega)=1+\frac{\psi_{r}(t, \omega)}{e^{-2 \pi i \omega} G_{r}(t, \omega)}
$$

Clearly $\delta_{r}$ is continuous. Also, using Theorem $6.5 \mathrm{~b}, \mathrm{~d}$, and the inequality $\left|G_{r}(t, \omega)\right| \geq A^{1 / 2} / 2$ that we verified at the beginning of this proof, we see that $\delta_{r}$ is nonvanishing on $Q$ for small enough $r$. Hence there is a continuous function $\theta_{r}(t, \omega)$ such that

$$
\delta_{r}(t, \omega)=\left|\delta_{r}(t, \omega)\right| e^{i \theta_{r}(t, \omega)} .
$$

Note that by Theorem $6.5 \mathrm{~d}, \delta_{r}$ converges uniformly to 1 on $\bar{Q}$ as $r \rightarrow 0$, and hence $\theta_{r}$ converges uniformly on $\bar{Q}$ to a multiple of $2 \pi$. Also note that by definition, 


$$
G_{r}(t+1, \omega)=e^{-2 \pi i \omega} G_{r}(t, \omega) \delta_{r}(t, \omega)
$$

for every $t$ and $\omega$.

Now, we have that

$$
\begin{aligned}
\left|G_{r}(1, \omega)\right| e^{i \varphi_{r}(1, \omega)}=G_{r}(1, \omega) & =e^{-2 \pi i \omega} G_{r}(0, \omega) \delta_{r}(0, \omega) \\
& =\left|G_{r}(0, \omega) \delta_{r}(0, \omega)\right| e^{-2 \pi i \omega+i \theta_{r}(0, \omega)+i \varphi_{r}(0, \omega)} \\
& =\left|G_{r}(1, \omega)\right| e^{-2 \pi i \omega+i \theta_{r}(0, \omega)+i \varphi_{r}(0, \omega)} .
\end{aligned}
$$

Hence for each $r>0$ there is an integer $l_{r}$ such that for all $\omega \in[0,1]$,

$$
\varphi_{r}(1, \omega)=-2 \pi \omega+\theta_{r}(0, \omega)+\varphi_{r}(0, \omega)+2 \pi l_{r} .
$$

Finally, we have

$$
\begin{aligned}
0= & \left(\varphi_{r}(0,0)-\varphi_{r}(1,0)\right)+\left(\varphi_{r}(1,0)-\varphi_{r}(1,1)\right) \\
& +\left(\varphi_{r}(1,1)-\varphi_{r}(0,1)\right)+\left(\varphi_{r}(0,1)-\varphi_{r}(0,0)\right) \\
= & \left(-\theta_{r}(0,0)-2 \pi l_{r}\right)+\left(-2 \pi k_{r}\right)+\left(-2 \pi+\theta_{r}(0,1)+2 \pi l_{r}\right)+\left(2 \pi k_{r}\right) \\
= & -2 \pi+\theta_{r}(0,1)-\theta_{r}(0,0) .
\end{aligned}
$$

Letting $r \rightarrow 0$, we find that $0=-2 \pi$, a contradiction.

\section{The Uncertainty Principle Approach to the BLT}

We shall present a proof of the BLT due to Daubechies and Janssen [DJ93], based on the operator theory associated with the Classical Uncertainty Principle Inequality and inspired by the elegant proof of the BLT for orthornormal bases by Battle [Bat88].

We shall use the basic properties of frames, Gabor systems, and the Zak transform discussed in $\S 2.1, \S 2.2$, and $\S 3.1$. In particular, we may consider only Gabor systems with $a=b=1$. In this case, $\left\{g_{m, n}\right\}$ is a frame for $L^{2}(\mathbf{R})$ if and only if it is an exact frame (Theorem 3.1d). Therefore the dual frame $\left\{\tilde{g}_{m, n}\right\}$ is biorthogonal to $\left\{g_{m, n}\right\}$, that is, $\left\langle g_{m, n}, \tilde{g}_{m^{\prime}, n^{\prime}}\right\rangle=\delta_{m, m^{\prime}} \delta_{n, n^{\prime}}$. Also, $Z g_{m, n}(t, \omega)=$ $e_{m}(t) e_{n}(\omega) Z g(t, \omega)$.

\subsection{Uncertainty Principles}

The Classical Uncertainty Principle Inequality was stated in Theorem 1.2. We prove it for $f \in \mathcal{S}(\mathbf{R}):$

$$
\begin{aligned}
\|f\|_{2}^{2}=\int t\left(|f|^{2}\right)^{\prime}(t) d t & \leq \int|t|\left(2\left|\overline{f(t)} f^{\prime}(t)\right|\right) d t \\
& \leq 2\|t f(t)\|_{2}\left\|f^{\prime}\right\|_{2} \\
& =4 \pi\|t f(t)\|_{2}\|\gamma \hat{f}(\gamma)\|_{2}
\end{aligned}
$$


A standard closure argument, e.g., [Ben94, Remark 7.31], which we also use in Lemma 7.2, extends the inequality to all $f \in L^{2}(\mathbf{R})$. There is a theory of weighted and local uncertainty principles emanating from Theorem 1.2 and its Hilbert space analogue, Theorem 7.1. This theory is the subject of [Ben94, Section 7.8], [Ben96, Chapter 6].

The Classical Uncertainty Principle Inequality can be formulated in general Hilbert spaces. Given a Hilbert space $H$ with inner product $\langle f, g\rangle$ and norm $\|f\|=\langle f, f\rangle^{1 / 2}$, and given operators $A$, $B$ mapping $H$ into $H$ (or mapping domains $D(A), D(B) \subseteq H$ into $H$ ), we define the commutator of $A$ and $B$ to be

$$
[A, B]=A B-B A .
$$

If $A$ is self-adjoint (i.e., $\langle A f, f\rangle=\langle f, A f\rangle$ for all $f \in H$ ), then the expectation of $A$ at $f \in D(A)$ is $E_{f}(A)=\langle A f, f\rangle$, and the variance of $A$ at $f \in D\left(A^{2}\right)$ is $\sigma_{f}^{2}(A)=E_{f}\left(A^{2}\right)-\left(E_{f}(A)\right)^{2}$.

\subsection{Theorem.}

Given self-adjoint (but not necessarily continuous) operators $A, B$ on a Hilbert space $H$. If $f \in D\left(A^{2}\right) \cap D\left(B^{2}\right) \cap D(i[A, B])$ and $\|f\|=1$, then

$$
E_{f}(i[A, B])^{2} \leq 4 \sigma_{f}^{2}(A) \sigma_{f}^{2}(B) .
$$

The proof of Theorem 7.1 is not difficult (for example, [Ben94, Theorem 7.32]) and we obtain the Classical Uncertainty Principle Inequality as a corollary, as follows. Define the position operator $P$ and momentum operator $M$ by

$$
P f(t)=t f(t) \quad \text { and } \quad M f=(P \hat{f})^{\vee}=(\gamma \hat{f}(\gamma))^{\vee},
$$

when these make sense. Both $P$ and $M$ are self-adjoint and if $f \in \mathcal{S}(\mathbf{R})$ then $\left(f^{\prime}\right)^{\wedge}=2 \pi i P \hat{f}$, $f^{\prime}=2 \pi i M f$, and $[P, M] f=-\frac{1}{2 \pi i} f$. By Theorem 7.1, we have

$$
E_{f}\left(-\frac{1}{2 \pi} I\right)^{2} \leq \sigma_{f}^{2}(P) \sigma_{f}^{2}(M)
$$

where $I$ is the identity operator. On the other hand, $E_{f}(I)=\langle I f, f\rangle=\|f\|_{2}^{2}$, and

$$
\sigma_{f}^{2}(P)=\|P f\|_{2}^{2}-\langle P f, f\rangle^{2} \leq\|P f\|_{2}^{2}=\|t f(t)\|_{2}^{2},
$$

and

$$
\sigma_{f}^{2}(M)=\|M f\|_{2}^{2}-\langle M f, f\rangle^{2} \leq\|M f\|_{2}^{2}=\|\gamma \hat{f}(\gamma)\|_{2}^{2},
$$

since both $\langle P f, f\rangle=\int t|f(t)|^{2} d t$ and $\langle M f, f\rangle=\int \gamma|\hat{f}(\gamma)|^{2} d \gamma$ are real, hence have nonnegative squares. The Classical Uncertainty Principle Inequality follows immediately. 


\subsection{The Weak BLT}

The fact that $[P, M]=-\frac{1}{2 \pi i} I$ forms the core of the uncertainty principle approach to proving the BLT. We state this fact in the following form.

\subsection{Lemma.}

If $f, g \in L^{2}(\mathbf{R})$ satisfy $P f, P g \in L^{2}(\mathbf{R})$ and $P \hat{f}, P \hat{g} \in L^{2}(\hat{\mathbf{R}})$, then

$$
\langle P f, M g\rangle-\langle M f, P g\rangle=\frac{1}{2 \pi i}\langle f, g\rangle .
$$

Proof. $M f$ and $M g$ are well-defined since $P \hat{f}, P \hat{g} \in L^{2}(\hat{\mathbf{R}})$. By standard techniques we can find $\varphi_{k}, \psi_{k} \in \mathcal{S}(\mathbf{R})$ such that $\varphi_{k} \rightarrow f, P \varphi_{k} \rightarrow P f, M \varphi_{k} \rightarrow M f$, and $\psi_{k} \rightarrow g, P \psi_{k} \rightarrow P g, M \psi_{k} \rightarrow M g$, all in $L^{2}$-norm. Since $\varphi_{k}, \psi_{k} \in \mathcal{S}(\mathbf{R})$ and $P, M$ are self-adjoint, we have

$$
\begin{aligned}
\left\langle P \varphi_{k}, M \psi_{k}\right\rangle-\left\langle M \varphi_{k}, P \psi_{k}\right\rangle & =\left\langle M P \varphi_{k}, \psi_{k}\right\rangle-\left\langle P M \varphi_{k}, \psi_{k}\right\rangle \\
& =-\left\langle[P, M] \varphi_{k}, \psi_{k}\right\rangle \\
& =\frac{1}{2 \pi i}\left\langle\varphi_{k}, \psi_{k}\right\rangle
\end{aligned}
$$

However, the inner product is continuous, so $\left\langle\varphi_{k}, \psi_{k}\right\rangle \rightarrow\langle f, g\rangle,\left\langle P \varphi_{k}, M \psi_{k}\right\rangle \rightarrow\langle P f, M g\rangle$, and $\left\langle M \varphi_{k}, P \psi_{k}\right\rangle \rightarrow\langle M f, P g\rangle$. Therefore (7.1) holds.

Next, we compute the commutators of $P$ and $M$ with the translation and modulation operators $\tau_{n}$ and $e_{m}$ defined in (2.3).

\subsection{Lemma.}

a. $\left[e_{m} \tau_{n}, P\right]=e_{m} \tau_{n} P-P e_{m} \tau_{n}=-n e_{m} \tau_{n}$.

b. $\left[e_{m} \tau_{n}, M\right]=e_{m} \tau_{n} M-M e_{m} \tau_{n}=-m e_{m} \tau_{n}$.

Proof. As the two parts are similar, we prove only part a. We compute

$$
\begin{aligned}
\left(e_{m} \tau_{n} P f\right)(t)-\left(P e_{m} \tau_{n} f\right)(t) & =e_{m}(t)\left(\tau_{n} P f\right)(t)-t\left(e_{m} \tau_{n} f\right)(t) \\
& =e_{m}(t)(P f)(t-n)-t e_{m}(t)\left(\tau_{n} f\right)(t) \\
& =e_{m}(t)(t-n) f(t-n)-t e_{m}(t) f(t-n) \\
& =-n e_{m}(t) f(t-n) \\
& =-n e_{m}(t)\left(\tau_{n} f\right)(t) \\
& =-n\left(e_{m} \tau_{n} f\right)(t) .
\end{aligned}
$$

We can now prove a weak version of the BLT. 


\subsection{Theorem (Weak BLT).}

Assume $g \in L^{2}(\mathbf{R})$ is such that $\left\{g_{m, n}\right\}$ is an exact frame for $L^{2}(\mathbf{R})$. Then we cannot have all of $P g, P \tilde{g} \in L^{2}(\mathbf{R})$ and $P \hat{g}, P \hat{\tilde{g}} \in L^{2}(\hat{\mathbf{R}})$, that is, we must have

$$
\|t g(t)\|_{2}\|\gamma \hat{g}(\gamma)\|_{2}\|t \tilde{g}(t)\|_{2}\|\gamma \hat{\tilde{g}}(\gamma)\|_{2}=+\infty
$$

Proof. Assume all four functions were elements of $L^{2}$. Note that

$$
\forall f, h \in L^{2}(\mathbf{R}), \quad\left\langle f, h_{m, n}\right\rangle=\left\langle f_{-m,-n}, h\right\rangle .
$$

Also, by Lemma 7.3a,

$$
\forall f \in L^{2}(\mathbf{R}), \quad P\left(f_{m, n}\right)=(P f)_{m, n}+n f_{m, n} .
$$

Since $P$ is self-adjoint and $\left\{g_{m, n}\right\}$ is biorthonormal to its dual frame $\left\{\tilde{g}_{m, n}\right\}$, we can therefore compute

$$
\begin{aligned}
\left\langle P g, \tilde{g}_{m, n}\right\rangle=\left\langle g, P\left(\tilde{g}_{m, n}\right)\right\rangle & \left.\left.=\left\langle g,(P \tilde{g})_{m, n}\right)\right\rangle+n\left\langle g, \tilde{g}_{m, n}\right)\right\rangle \\
& =\left\langle g_{-m,-n}, P \tilde{g}\right\rangle+n \delta_{m, 0} \delta_{n, 0} \\
& =\left\langle g_{-m,-n}, P \tilde{g}\right\rangle .
\end{aligned}
$$

Now, by the $L^{2}$-inversion formula, both $M g$ and $M \tilde{g}$ exist and are in $L^{2}(\mathbf{R})$, so by Lemma $7.3 \mathrm{~b}$ we similarly obtain

$$
\left\langle g_{m, n}, M \tilde{g}\right\rangle=\left\langle M\left(g_{m, n}\right), \tilde{g}\right\rangle=\left\langle(M g)_{m, n}, \tilde{g}\right\rangle+m\left\langle g_{m, n}, \tilde{g}\right\rangle=\left\langle M g, \tilde{g}_{-m,-n}\right\rangle .
$$

Since $f=\sum\left\langle f, g_{m, n}\right\rangle \tilde{g}_{m, n}=\sum\left\langle f, \tilde{g}_{m, n}\right\rangle g_{m, n}$ for every $f \in L^{2}(\mathbf{R})$, we therefore have

$$
\begin{aligned}
\langle P g, M \tilde{g}\rangle & =\sum_{m, n}\left\langle P g, \tilde{g}_{m, n}\right\rangle\left\langle g_{m, n}, M \tilde{g}\right\rangle \\
& =\sum_{m, n}\left\langle g_{-m,-n}, P \tilde{g}\right\rangle\left\langle M g, \tilde{g}_{-m,-n}\right\rangle \\
& =\sum_{m, n}\left\langle M g, \tilde{g}_{m, n}\right\rangle\left\langle g_{m, n}, P \tilde{g}\right\rangle \\
& =\langle M g, P \tilde{g}\rangle .
\end{aligned}
$$

Therefore, by biorthonormality and Lemma 7.2,

$$
1=\langle g, \tilde{g}\rangle=2 \pi i(\langle P g, M \tilde{g}\rangle-\langle M g, P \tilde{g}\rangle)=0,
$$

a contradiction. 


\subsection{Equivalence of the weak BLT and the BLT}

We can give several special cases illustrating the relationship between the weak BLT and the usual BLT (Theorem 1.1).

7.5. Example. a. If the Gabor frame $\left\{g_{m, n}\right\}$ is actually an orthonormal basis, then $\tilde{g}=g$ and the equivalence is clear. This is precisely Battle's proof of the BLT [Bat88].

b. If $g$ generates a tight exact frame with bounds $A=B$ then $\tilde{g}=S^{-1} g=A^{-1} g$, and the equivalence is again clear. However, any tight exact frame is a multiple of an orthonormal basis.

c. If $\operatorname{supp}(g)$ is contained in an interval of length 1, then the frame operator $S$ is $S f=f \cdot \lambda$, where $\lambda(t)=\sum|g(t-n)|^{2}$ (for example, [HW89]). Any Gabor frame must have $A \leq \lambda(t) \leq B$ a.e. (Theorem 3.5), so $S$ is multiplication by an essentially constant function. Therefore, $\tilde{g}=S^{-1} \tilde{g}=g / \lambda$, and thus $P g \in L^{2}(\mathbf{R})$ if and only if $P \tilde{g} \in L^{2}(\mathbf{R})$. Similarly, if $\operatorname{supp}(\hat{g})$ is contained in an interval of length 1 , then $(S f)^{\wedge}=\hat{f} \cdot \Lambda$, where $\Lambda$ is the essentially constant function $\sum|\hat{g}(\gamma-m)|^{2}$, and so $P \hat{g} \in L^{2}(\hat{\mathbf{R}})$ if and only if $P \hat{\tilde{g}} \in L^{2}(\hat{\mathbf{R}})$.

The BLT will follow from the weak BLT if we can prove that

$$
P g \in L^{2}(\mathbf{R}) \Leftrightarrow P \tilde{g} \in L^{2}(\mathbf{R}) \quad \text { and } \quad P \hat{g} \in L^{2}(\hat{\mathbf{R}}) \Leftrightarrow P \hat{\tilde{g}} \in L^{2}(\hat{\mathbf{R}}),
$$

whenever $\left\{g_{m, n}\right\}$ is an exact frame. We verify (7.2) in Theorem 7.7. First, however, we compute the Zak transform of the dual function $\tilde{g}$.

\subsection{Proposition.}

If $g \in L^{2}(\mathbf{R})$ and $\left\{g_{m, n}\right\}$ is a frame then

$$
Z \tilde{g}=1 / \overline{Z g}
$$

Proof. If $\left\{g_{m, n}\right\}$ is a frame then $0<A \leq|Z g|^{2} \leq B<\infty$ a.e. on $Q$. Therefore, $h=Z^{-1}(1 / \overline{Z g}) \in$ $L^{2}(\mathbf{R})$. Given $m, n \in \mathbf{Z}$ we then compute:

$$
\begin{aligned}
\left\langle h, g_{m, n}\right\rangle=\left\langle Z h, Z g_{m, n}\right\rangle & =\left\langle 1 / \overline{Z g}, e_{m}(t) e_{n}(\omega) Z g\right\rangle \\
& =\left\langle 1, e_{m}(t) e_{n}(\omega)\right\rangle \\
& =\delta_{m, 0} \delta_{n, 0} \\
& =\left\langle\tilde{g}, g_{m, n}\right\rangle .
\end{aligned}
$$

Since $\left\{g_{m, n}\right\}$ is complete in $L^{2}(\mathbf{R})$ and $h, \tilde{g} \in L^{2}(\mathbf{R})$, it follows that $h=\tilde{g}$.

The following theorem is due to Daubechies and Janssen [DJ93].

\subsection{Theorem.}

If $\left\{g_{m, n}\right\}$ is an exact frame then (7.2) holds. 
Proof. First, given any function $f$, we formally compute:

$$
\begin{aligned}
Z P f(t, \omega) & =\sum_{k} P f(t+k) e^{2 \pi i k \omega} \\
& =t \sum_{k} f(t+k) e^{2 \pi i k \omega}+\sum_{k} f(t+k) k e^{2 \pi i k \omega} \\
& =t Z f(t, \omega)+\sum_{k} f(t+k) \frac{1}{2 \pi i} \partial_{2} e^{2 \pi i k \omega} \\
& =t Z f(t, \omega)+\frac{1}{2 \pi i} \partial_{2} Z f(t, \omega) .
\end{aligned}
$$

Next, using Proposition 7.6, we formally compute:

$$
\begin{aligned}
\overline{Z P \tilde{g}(t, \omega)} & =t \overline{Z \tilde{g}(t, \omega)}+\overline{\frac{1}{2 \pi i} \partial_{2} Z \tilde{g}(t, \omega)} \\
& =\frac{t}{Z g(t, \omega)}-\frac{1}{2 \pi i} \partial_{2}(1 / Z g)(t, \omega) \\
& =\frac{t}{Z g(t, \omega)}+\frac{\frac{1}{2 \pi i} \partial_{2} Z g(t, \omega)}{Z g(t, \omega)^{2}} \\
& =\frac{t Z g(t, \omega)+\frac{1}{2 \pi i} \partial_{2} Z g(t, \omega)}{Z g(t, \omega)^{2}} \\
& =\frac{Z P g(t, \omega)}{Z g(t, \omega)^{2}}
\end{aligned}
$$

If these formal calculations are justified, then $Z P g \in L^{2}(Q)$ if and only if $Z P \tilde{g} \in L^{2}(Q)$ since $0<A \leq|Z g|^{2} \leq B<\infty$ a.e. As $Z$ is unitary, this implies $P g \in L^{2}(\mathbf{R})$ if and only if $P \tilde{g} \in L^{2}(\mathbf{R})$. A similar formal argument involving the partial derivative $\partial_{1}$ is used to show that $P \hat{g} \in L^{2}(\hat{\mathbf{R}})$ if and only if $P \hat{\tilde{g}} \in L^{2}(\hat{\mathbf{R}})$. The critical step is the use of the quotient rule in (7.4).

The calculations above are justified as follows. If $f \in L^{2}(\mathbf{R})$ then (7.3) is valid distributionally, that is, in the $\sigma\left(D^{\prime}\left(\mathbf{R}^{2}\right), C_{c}^{\infty}\left(\mathbf{R}^{2}\right)\right)$ topology. In particular, (7.3) is true distributionally for $f=g$ and $f=\tilde{g}$. Then (7.4) follows from Theorem 5.1a. In fact, $G=Z g \in A C_{\text {loc }}$ on almost all straight lines parallel to the coordinate axes since $\partial_{1} G, \partial_{2} G \in L_{\text {loc }}^{2}\left(\mathbf{R}^{2}\right)$ (for example, Example 5.3a). Then, for almost all $t$, the quotient rule to compute the classical partial derivative $D_{2}(1 / G)$ holds for almost all $\omega$. Distributional and classical differentiation are equivalent in this case by Theorem 5.1a. Therefore (7.4) is valid distributionally, and so the distributions $\overline{Z P \tilde{g}}$ and $Z P g /(Z g)^{2}$ are equal. Thus, if one of them is square integrable then so is the other.

The combination of the Weak BLT (Theorem 7.4) and Theorem 7.7 proves the BLT. 


\subsection{The BLT without differentiation}

It is interesting to recount that distributional differentiation really initiated the proof of the BLT in [Dau90] before shifting to classical differentiation, e.g., Example 5.3a,b; and that, in the proof of the BLT in Theorem 7.7, classical differentiation was required to implement the quotient rule in order to proceed with distributional calculations. In both cases, Theorem 5.1a played a pivotal role. Of course, neither classical nor distributional differentiation was overtly involved in the proof of the BLT in $\S 6$.

The final issue we wish to address is the possibility of proving the BLT for exact frames along the operator-theoretic lines of $\S 7.2$, but without invoking differentiation. The proof of Proposition 7.6 motivates the argument we have in mind. Let us attempt to use the same technique to prove that $P g \in L^{2}(\mathbf{R})$ if and only if $P \tilde{g} \in L^{2}(\mathbf{R})$. Assume that $g \in L^{2}(\mathbf{R})$ generates an exact frame $\left\{g_{m, n}\right\}$ and that $P g \in L^{2}(\mathbf{R})$. Then $Z P g \in L^{2}(Q)$ and $Z g$ is essentially constant, so $Z P g /(Z g)^{2} \in L^{2}(Q)$. Therefore, $h=Z^{-1}\left(\overline{Z P g /(Z g)^{2}}\right) \in L^{2}(\mathbf{R})$. Note that

$$
\left\langle h, g_{m, n}\right\rangle=\left\langle Z h, Z g_{m, n}\right\rangle=\left\langle\overline{Z P g /(Z g)^{2}}, e_{m}(t) e_{n}(\omega) Z g\right\rangle=\left\langle\overline{Z P g / Z g}, e_{m}(t) e_{n}(\omega)\right\rangle .
$$

By Lemma 7.3a, $P g_{m, n}=(P g)_{m, n}+n g_{m, n} \in L^{2}(\mathbf{R})$, so we can compute

$$
\begin{aligned}
\left\langle\tilde{g}, P g_{m, n}\right\rangle & =\left\langle\tilde{g},(P g)_{m, n}\right\rangle+n\left\langle\tilde{g}, g_{m, n}\right\rangle \\
& =\left\langle Z \tilde{g}, Z(P g)_{m, n}\right\rangle+n \delta_{m, 0} \delta_{n, 0} \\
& =\left\langle 1 / \overline{Z g}, e_{m}(t) e_{n}(\omega) Z(P g)\right\rangle \\
& =\left\langle h, g_{m, n}\right\rangle .
\end{aligned}
$$

Although we have not assumed that $P \tilde{g} \in L^{2}(\mathbf{R})$, the integral $\int P \tilde{g}(t) g_{m, n}(t) d t$ is well-defined since it equals $\int \tilde{g}(t) P g_{m, n}(t) d t$ and $\tilde{g} \cdot P g \in L^{1}(\mathbf{R})$. Thus,

$$
\left\langle P \tilde{g}-h, g_{m, n}\right\rangle=\left\langle\tilde{g}, P g_{m, n}\right\rangle-\left\langle h, g_{m, n}\right\rangle=0
$$

for all $m, n \in \mathbf{Z}$. We would like to conclude from (7.5) and the fact that $\left\{g_{m, n}\right\}$ is complete in $L^{2}(\mathbf{R})$ that $P g-h=0$. Note that the function $f=P \tilde{g}-h=f_{1}+f_{2}$ has the property that $f_{1} \in t L^{2}(\mathbf{R})$ and $f_{2} \in L^{2}(\mathbf{R})$.

Problem/Examples 7.8. Motivated by the preceding discussion, we pose the following uniqueness problem. Let $f \in L_{\mathrm{loc}}^{2}(\mathbf{R})$ and assume that $\left\{g_{k}\right\}$ is a complete sequence in $L^{2}(\mathbf{R})$. Suppose that $f \overline{g_{k}} \in L^{1}(\mathbf{R})$ and that $\int f(t) \overline{g_{k}(t)} d t=0$ for all $k$. What further conditions must be assumed to conclude that $f=0$ ?

The following examples deal with our uniqueness problem.

a. The Haar system $\left\{h_{k}\right\} \subseteq L^{2}(\mathbf{R})$ is an orthonormal basis of $L^{2}(\mathbf{R})$; and if $f=1$, then $f \overline{h_{k}} \in L^{1}(\mathbf{R})$ and $\left\langle f, h_{k}\right\rangle=0$ for all $k$. In fact, this holds for any wavelet orthonormal basis whose elements are integrable.

b. Uniqueness also fails for the Gabor sequence $\left\{g_{m, n}\right\}$ generated by $g=\mathbf{1}_{[0,1]}-\mathbf{1}_{[-1,0]}$. In fact, if $f=1$, then $f \overline{g_{m, n}} \in L^{1}(\mathbf{R})$ and $\left\langle f, g_{m, n}\right\rangle=0$ for all $m$ and $n$. Further, the Zak transform of $g$ is 


$$
Z g(t, \omega)=1-e^{-2 \pi i \omega} \neq 0 \text { a.e. on } Q
$$

so that $\left\{g_{m, n}\right\} \subseteq L^{2}(\mathbf{R})$ is a complete sequence (for example, Theorem 3.1a). In contrast to part a, $\left\{g_{m, n}\right\}$ is not even a frame.

c. Let $g=\sum a_{k} \mathbf{1}_{[k-1 / 2, k+1 / 2]}$ where $\sum\left|a_{k}\right|<\infty$ and $\sum a_{k}=0$. Then $Z g(t, \omega)=\sum a_{k} e^{2 \pi i k \omega}$ for $t \in(-1 / 2,1 / 2)$, so that $Z g$ is continuous in a neighborhood of $(0,0)$ and $Z g(0,0)=0$. Thus $\left\{g_{m, n}\right\}$ is not a Gabor frame. On the other hand, if $f=1$ then $f \overline{g_{m, n}} \in L^{1}(\mathbf{R})$ and $\left\langle f, g_{m, n}\right\rangle=0$ for all $m$ and $n$.

d. Let $g \in L^{2}(\mathbf{R}) \backslash\left(L^{1}(\mathbf{R}) \cap L^{2}(\mathbf{R})\right)$ be the odd function defined as $g=0$ on $[0,1 / 2), g=0$ on $[2 k-1,2 k+1)$ for $k$ even, and $g=-2 i /(\pi k)$ on $[2 k-1,2 k+1)$ for $k$ odd. We can write $g$ as

$$
g(t)=g(u-k)=\int_{-1 / 2}^{1 / 2} G(u, \omega) e^{-2 \pi i k \omega} d \omega,
$$

where $t=u-k, u \in[-1 / 2,1 / 2), k \in \mathbf{Z}$, and $G(u, \omega)$ is 1 on $[-1 / 2,1 / 2) \times[0,1 / 2)$ and is -1 on $[-1 / 2,1 / 2) \times[-1 / 2,0)$. Thus, $G=Z g$ and $\left\{g_{m, n}\right\}$ is a Gabor frame. If $f=1$ then $\left\langle f, g_{m, n}\right\rangle=0$ for all $m$ and $n$, whereas $f \overline{g_{m, n}} \notin L^{1}(\mathbf{R})$. Clearly, $P g \notin L^{2}(\mathbf{R})$.

\section{Appendix: Notation}

The integral over $\mathbf{R}^{d}$ (usually $d=1,2$ in this paper) is designated by " $\int$ ". Sequences with unspecified limits are to be taken over the set of integers $\mathbf{Z}$.

The space $L^{p}\left(\mathbf{R}^{d}\right)$ consists of all functions $f$ such that $\|f\|_{p}<\infty$, where $\|f\|_{p}=\left(\int|f(t)|^{p} d t\right)^{1 / p}$ if $1 \leq p<\infty$ and $\|f\|_{\infty}=\operatorname{ess} \sup |f(t)|$. The inner product of $f, g \in L^{2}\left(\mathbf{R}^{d}\right)$ is $\langle f, g\rangle=\int f(t) \overline{g(t)} d t$, where the bar denotes complex conjugation.

The Fourier transform of $f \in L^{1}(\mathbf{R})$ is $\hat{f}(\gamma)=\int f(t) e^{-2 \pi i \gamma t} d t$ for $\gamma \in \hat{\mathbf{R}}$. The inverse Fourier transform is $\check{f}(\gamma)=\hat{f}(-\gamma)$. The Fourier transform extends to $f \in L^{2}(\mathbf{R})$. If $f \in L^{2}(\mathbf{R})$ then $f=(\hat{f})^{\vee}$.

$C\left(\mathbf{R}^{d}\right)$ is the space of continuous functions on $\mathbf{R}^{d}$. $C_{c}\left(\mathbf{R}^{d}\right)$ is the space of continuous functions with compact support. $C_{c}^{\infty}\left(\mathbf{R}^{d}\right)$ is the space of compactly supported functions infinitely differentiable on $\mathbf{R}^{d} . C_{0}\left(\mathbf{R}^{d}\right)$ is the space of continuous functions on $\mathbf{R}^{d}$ vanishing at infinity. $\mathcal{S}\left(\mathbf{R}^{d}\right)$ is the Schwartz class of infinitely differentiable functions which are rapidly decreasing at infinity, and $\mathcal{S}^{\prime}\left(\mathbf{R}^{d}\right)$ is its topological dual, the space of tempered distributions. $A\left(\hat{\mathbf{R}}^{d}\right)$ is $L^{1}\left(\mathbf{R}^{d}\right)^{\wedge}=\{\hat{f}: f \in$ $\left.L^{1}\left(\mathbf{R}^{d}\right)\right\} . A C_{\text {loc }}$ is the space of all functions that are locally absolutely continuous.

If $g \in L^{2}(\mathbf{R})$ and $a, b>0$ are given then the Gabor system generated by $g, a$, and $b$ is $\left\{g_{m b, n a}\right\}_{m, n \in \mathbf{Z}}$, where $g_{p, q}(t)=e^{2 \pi i p t} g(t-q)$. If $\left\{g_{m b, n a}\right\}$ is a frame then the frame operator is $S f=\sum\left\langle f, g_{m b, n a}\right\rangle g_{m b, n a}$. The dual function is $\tilde{g}=S^{-1} g$. The dual frame is $\left\{\tilde{g}_{m b, n a}\right\}$.

Distributional differentiation is denoted by $\partial$ on $\mathbf{R}$ or $\partial_{j}$ on $\mathbf{R}^{d}$ (the $j$-th partial). Classical differentiation is denoted by $D$ on $\mathbf{R}$ and $D_{j}$ on $\mathbf{R}^{d}$. The Sobolev space $L_{1}^{2}\left(\mathbf{R}^{d}\right)$ is $\left\{f \in L^{2}\left(\mathbf{R}^{d}\right)\right.$ : $\left.\partial_{j} f \in L^{2}\left(\mathbf{R}^{d}\right), j=1, \ldots, d\right\}$.

If $x=(t, \omega) \in \mathbf{R} \times \hat{\mathbf{R}}$ and $r>0$ then $Q(x ; r)=Q(t, \omega ; r)$ is the square centered at $x$ with side $r$, i.e., $Q(x ; r)=[t-r / 2, t+r / 2) \times[\omega-r / 2, \omega+r / 2)$. We set $Q=Q(1 / 2,1 / 2 ; 1)=[0,1) \times[0,1)$.

The translation operator is $\tau_{x} f(t)=f(t-x)$ and the modulation operator is $e_{\gamma} f(t)=$ $e^{2 \pi i \gamma t} f(t)$. We also write $e_{\gamma}(t)=e^{2 \pi i \gamma t}$. 
The indicator function of the set $E$ is $\mathbf{1}_{E}(x)=1$ if $x \in E$ and 0 if $x \notin E$. The Kronecker delta is $\delta_{k, \ell}=1$ if $k=\ell$ and 0 if $k \neq \ell$.

\section{References}

[AGT92] L. Auslander, I. Gertner, and R. Tolimieri, The finite Zak transform and the finite Fourier transform, Radar and Sonar, Part II (F. A. Grunbaum, M. Bernfeld, and R. E. Blahut, eds.), IMA Vol. Math. Appl., vol. 39, Springer-Verlag, New York, 1995, pp. 21-35.

[AT75] L. Auslander and R. Tolimieri, Abelian Harmonic Analysis, Theta Functions and Function Algebras on a Nilmanifold, Lecture Notes in Math., vol. 436, Springer-Verlag, New York, 1975.

[Aus94] P. Auscher, Remarks on the local Fourier bases, Wavelets: Mathematics and Applications (J. J. Benedetto and M. W. Frazier, eds.), CRC Press, Boca Raton, FL, 1993, pp. 203-218.

[AWW92] P. Auscher, G. Weiss, and M. V. Wickerhauser, Local sine and cosine basis of Coifman and Meyer and the construction of smooth wavelets, Wavelets: A Tutorial in Theory and Applications (C. K. Chui, ed.), Academic Press, Boston, 1992, pp. 273-256.

[BGZ75] H. Bacry, A. Grossmann, and J. Zak, Proof of completeness of lattice states in the kq representation, Phys. Rev. B 12 (1975), 1118-1120.

[Bal81] R. Balian, Un principe d'incertitude fort en théorie du signal ou en mécanique quantique, C. R. Acad. Sci. Paris 292 (1981), 1357-1362.

[Bar51] N. Bari, Biorthogonal systems and bases in Hilbert space, Učen Zap. Mosk. Gos. Univ. 148, Mat. 4 (1951), 69-107.

[Bat88] G. Battle, Heisenberg proof of the Balian-Low theorem, Lett. Math. Phys. 15 (1988), 175-177.

[Bat89] _ Phase space localization theorem for ondelettes, J. Math. Phys. 30 (1989), 2195-2196.

[Ben76] J. J. Benedetto, Real Variable and Integration, B. G. Teubner, Stuttgart, 1976.

[Ben94] Frame decompositions, sampling, and uncertainty principle inequalities, Wavelets: Mathematics and Applications (J. J. Benedetto and M. W. Frazier, eds.), CRC Press, Boca Raton, FL, 1993, pp. 247304.

[Ben96] Harmonic Analysis and Applications, CRC Press, Boca Raton, FL, 1996.

[BBE89] J. J. Benedetto, G. Benke, and W. Evans, An n-dimensional Wiener-Plancherel formula, Adv. in Appl. Math. 10 (1989), 457-487.

[BF94] J. J. Benedetto and M. W. Frazier, Wavelets: Mathematics and Applications (J. J. Benedetto and M. W. Frazier, eds.), CRC Press, Boca Raton, FL, 1994.

[BT93] J. J. Benedetto and A. Teolis, A wavelet auditory model and data compression, Appl. Comp. Harmonic Anal. 1 (1993), 3-28.

[BW94] J. J. Benedetto and D. Walnut, Gabor frames for $L^{2}$ and related spaces, Wavelets: Mathematics and Applications (J. J. Benedetto and M. W. Frazier, eds.), CRC Press, Boca Raton, FL, 1993, pp. 97-162.

[BM62] A. Beurling and P. Malliavin, On Fourier transforms of measures with compact support, Acta Math. 107 (1962), 291-309.

[BM67]_ On the closure of characters and the zeros of entire functions, Acta Math. 118 (1967), 79-93. 
[BZ81] M. Boon and J. Zak, Amplitudes on von Neumann lattices, J. Math. Phys. 22 (1981), 1090-1099.

[CH94] O. Christensen and C. Heil, Perturbations of Banach frames and atomic decompositions, preprint (1994).

[CS93] C. K. Chui, and X. Shi, Inequalities of Littlewood-Paley type for frames and wavelets, SIAM J. Math. Anal. 24 (1993), 263-277.

[CM91] R. R. Coifman, and Y. Meyer, Remarques sur l'analyse de Fourier à fenêtre, C. R. Acad. Sci, sér. 1312 (1991), 259-261.

[Dau86] I. Daubechies, Discrete sets of coherent states and their use in signal analysis, Proceedings of the International Conference on Differential Equations and Mathematical Physics, Birmingham, Alabama, March, 1986.

[Dau90] _ The wavelet transform, time-frequency localization and signal analysis, IEEE Trans. Inform. Theory 39 (1990), 961-1005.

[Dau92] _ Ten Lectures on Wavelets, SIAM Press, Philadelphia, 1992.

[DLL95] I. Daubechies, H. Landau, and Z. Landau, Gabor time-frequency lattices and the Wexler-Raz identity, J. Fourier Anal. Appl. (1995), this issue.

[DGM86] I. Daubechies, A. Grossmann, and Y. Meyer, Painless nonorthogonal expansions, J. Math. Phys. 27 (1986), 1271-1283.

[DJJ91] I. Daubechies, S. Jaffard, and J.-L. Journé, A simple Wilson orthonormal basis with exponential decay, SIAM J. Math. Anal. 22 (1991), 554-572.

[DJ93] I. Daubechies and A. J. E. M. Janssen, Two theorems on lattice expansions, IEEE Trans. Inform. Theory 39 (1993), 3-6.

[DS52] R. J. Duffin and A. C. Schaeffer, A class of nonharmonic Fourier series, Trans. Amer. Math. Soc. 72 (1952), 341-366.

[Fei89] H. Feichtinger, Atomic decompositions of modulation spaces, Rocky Mt. J. Math. 19 (1989), 113-126.

[Fei90] Generalized amalgams, with applications to Fourier transform, Canad. J. Math. 42 (1990), $394-409$.

[FG89] H. G. Feichtinger and K. Gröchenig, Banach spaces related to integrable group representations and their atomic decompositions, I, J. Funct. Anal. 86 (1989), 307-340; Banach spaces related to integrable group representations and their atomic decompositions, II, Monatshefte für Mathematik 108 (1989), 129-148.

[FGW92] H. G. Feichtinger, K. Gröchenig, and D. Walnut, Wilson bases and modulation spaces, Math. Nachr. 155 (1992), 7-17.

[FS85] J. Fournier and J. Stewart, Amalgams of $L^{p}$ and $\ell^{q}$, Bull. Amer. Math. Soc. (N.S.) 13 (1985), 1-21.

[Gab46] D. Gabor, Theory of communications, J. Inst. Elec. Eng. (London) 93 (1946), 429-457.

[Grö91] K. Gröchenig, Describing functions: Atomic decompositions versus frames, Monatshefte für Mathematik 112 (1991), 1-41.

[Grö93] _ Acceleration of the frame algorithm, IEEE Trans. Signal Proc. 41 (1993), 3331-3340.

[GW92] K. Gröchenig and D. Walnut, A Riesz basis for Bargmann-Fock space related to sampling and interpolation, Ark. Mat. 30 (1992), 283-295.

[Hei89] C. Heil, A discrete Zak transform, The MITRE Corporation, Technical Report MTR-89W00128 (1989).

[Hei90] Wiener amalgam spaces in generalized harmonic analysis and wavelet theory, Ph.D. thesis, University of Maryland, College Park, MD, 1990. 
[HRT94] C. Heil, J. Ramanathan, and P. Topiwala, Asymptotic singular value decay of time-frequency localization operators, Wavelet Applications in Signal and Image Processing II (A. F. Laine and M. A. Unser, eds.), vol. 2303, Society of Photo-Optical Industrial Engineers, Bellingham, WA, 1994, pp. 15-24.

[HRT95] _ Linear independence of time-frequency translates, Proc. Amer. Math. Soc. (1995) (to appear).

[HW89] C. Heil and D. Walnut, Continuous and discrete wavelet transforms, SIAM Review 31 (1989), 628-666.

[Hol94] J. R. Holub, Pre-frame operators, Besselian frames, and near-Riesz bases in Hilbert spaces, Proc. Amer. Math. Soc. 122 (1994), 779-785.

[Hör83] L. Hörmander, The Analysis of Linear Partial Differential Operators, Volumes I and II, Springer-Verlag, New York, 1983.

[Jan82] A. J. E. M. Janssen, Bargmann transform, Zak transform, and coherent states, J. Math. Phys. 23 (1982), $720-731$.

[Jan88] _ The Zak transform: a signal transform for sampled time-continuous signals, Philips J. Res. 43 (1988), 23-69.

[Jan94] _ Signal analytic proofs of two basic results on lattice expansions, Appl. Comp. Harm. Anal. 1 (1994), 350-354.

[Jan95] Duality and biorthogonality for Weyl-Heisenberg frames, J. Fourier Anal. Appl. (1995), this issue.

[JHJ88] H. Jensen, T. Høholdt, and J. Justesen, Double series representation of bounded signals, IEEE Trans. Inform. Theory 34 (1988), 613-624.

[Köt36] G. Köthe, Das Trägheitsgesetz der quadratischen Formen im Hilbertschen Raum, Math. Zeit. 41 (1936), 137-152.

[Lae90] E. Laeng, Une base orthonormale de $L^{2}(\mathbf{R})$ dont les éléments sont bien localisés dans l'espace de phase et leurs supports adaptés à toute partition symétrique de l'espaces des frèquencies, C. R. Acad. Sci., sér. 2311 (1990), 677-680.

[Lan64] H. J. Landau, A sparse regular sequence of exponentials closed on large sets, Bull. Amer. Math. Soc. 70 (1964), 566-569.

[Lan67] N Necessary density conditions for sampling and interpolation of certain entire functions, Acta Math. 117 (1967), 37-52.

[Lan93] _ On the density of phase-space expansions, IEEE Trans. Inform. Th. 39 (1993), 1152-1156.

[Lev40] N. Levinson, Gap and Density Theorems, AMS Colloquium Publications, vol. 26, American Mathematical Society, Providence, RI, 1940.

[Li94] S. Li, General frame decompositions, pseudo-duals and its applications to Weyl-Heisenberg frames, Numerical Optimization and Functional Analysis (to appear).

[LH95] S. Li and D. Healy, A parametric class of discrete Gabor expansions, preprint (1995).

[Low85] F. Low, Complete sets of wave packets, A Passion for Physics-Essays in Honor of Geoffrey Chew (C. DeTar, et al., eds.), World Scientific, Singapore, 1985, pp. 17-22.

[Mal90] H. Malvar, Lapped transform for efficient transform/subband coding, IEEE Trans. ASSP 38 (1990), 969978.

[Maz85] V. Maz’ja, Sobolev spaces, Springer-Verlag, New York, 1985.

[PW34] R. E. A. C. Paley, and N. Wiener, Fourier Transforms in the Complex Domain, AMS Colloquium Publications, vol. 19, American Mathematical Society, Providence, RI, 1934. 
[QCL92] S. Qian, K. Chen, and S. Li, Optimal biorthogonal functions for finite discrete-time Gabor expansions, Signal Processing 27 (1992), 177-185.

[RR55] T. Rado and P. Reichelderfer, Continuous Transformations in Analysis, Springer-Verlag, New York, 1955.

[RaS95] J. Ramanathan and T. Steger, Incompleteness of Sparse Coherent States, Appl. Comp. Harm. Anal. 2 (1995) (to appear).

[Rie81] M. Rieffel, Von Neumann algebras associated with pairs of lattices in Lie groups, Math. Ann. 257 (1981), 403-418.

[RoS94] A. Ron and Z. Shen, Weyl-Heisenberg frames and Riesz bases in $L_{2}\left(\mathbf{R}^{d}\right)$, preprint (1994).

[Sch66] L. Schwartz, Théorie des Distributions, Hermann, Paris, 1966.

[Sei92a] K. Seip, Sampling and interpolation in the Bargmann-Fock space, I, J. Reine Angew. Math. 429 (1992), 91-106.

[Sei92b] Wavelet in $H^{2}(\mathbf{R})$ : Sampling, interpolation, and phase space density, Wavelets-A Tutorial in Theory and Applications (C. K. Chui, eds.), Academic Press, New York, 1992, pp. 529-540.

[SeW92] K. Seip and R. Wallstén, Sampling and interpolation in the Bargmann-Fock space, II, J. Reine Angew. Math. 429 (1992), 107-113.

[Ste70] E. M. Stein, Singular Integrals and Differentiability Properties of Functions, Princeton University Press, Princeton, NJ, 1970.

[StW71] E. Stein and G. Weiss, Introduction to Fourier Analysis on Euclidean Spaces, Princeton University Press, Princeton, NJ, 1971.

[SRWW87] D. J. Sullivan, J. J. Rehr, J. W. Wilkins, and K. G. Wilson, Phase space Wannier functions in electronic structure calculations, preprint, Cornell University (1987).

[TO95] R. Tolimieri and R. S. Orr, Poisson summation, the ambiguity function, and the theory of WeylHeisenberg frames, J. Fourier Anal. Appl. (1995) (to appear).

[vN55] J. von Neumann, Mathematical Foundations of Quantum Mechanics, Princeton University Press, Princeton, NJ, 1932, 1949, 1955.

[Wal89a] D. Walnut, Weyl-Heisenberg wavelet expansions: existence and stability in weighted spaces, Ph.D. thesis, University of Maryland, College Park, MD, 1989.

[Wal89b] Wavelets in Discrete Domains, The MITRE Corporation, Technical Report MP-89W00040 (1989).

[Wal92] _ Continuity properties of the Gabor frame operator, J. Math. Anal. Appl. 165 (1992), 479-504.

[Wal93] _ Lattice size estimates for Gabor decompositions, Monatshefte für Mathematik 115 (1993), 245256.

[WR90] J. Wexler and S. Raz, Discrete Gabor expansions, Signal Processing 21 (1990), 207-220.

[Wil87] K. G. Wilson, Generalized Wannier functions, preprint, Cornell University (1987).

[You80] R. Young, An Introduction to Nonharmonic Fourier Series, Academic Press, New York, 1980.

[Zak75] J. Zak, Lattice operators in crystals for Bravais and reciprocal vectors, Phys. Rev. B 12 (1975), 3023-3026.

[ZZ93] M. Zibulski and Y. Y. Zeevi, Oversampling in the Gabor scheme, IEEE Trans. Signal Proc. 41 (1993), $2679-2687$. 
Received September 13, 1993

Department of Mathematics, University of Maryland, College Park, Maryland 20742 and The MITRE Corporation, McLean, Virginia 22102 (jjb@math.umd.edu)

School of Mathematics, Georgia Institute of Technology, Atlanta, Georgia 30332-0160 and The MITRE Corporation, Bedford, Massachusetts 01730 (heil@math.gatech.edu)

Department of Mathematics, George Mason University, Fairfax, Virginia 22030 (dwalnut@gmu.edu) 